PDES, SUBMANIFOLDS AND

AFFINE DIFFERENTIAL GEOMETRY

BANACH CENTER PUBLICATIONS, VOLUME 57

INSTITUTE OF MATHEMATICS

POLISH ACADEMY OF SCIENCES

WARSZAWA 2002

\title{
NATURAL ALGEBRAIC REPRESENTATION FORMULAS FOR CURVES IN $\mathbb{C}^{3}$
}

\author{
HUBERT GOLLEK \\ Institute of Mathematics, Humboldt University \\ Rudower Chaussee 25, 10099 Berlin, Germany \\ E-mail: gollek@mathematik.hu-berlin.de
}

\begin{abstract}
We consider several explicit examples of solutions of the differential equation $\Phi_{1}^{\prime 2}(z)+\Phi_{2}^{\prime 2}(z)+\Phi_{3}^{\prime 2}(z)=d^{2}(z)$ of meromorphic curves in $\mathbb{C}^{3}$ with preset infinitesimal arclength function $d(z)$ by nonlinear differential operators of the form $(f, h, d) \rightarrow \mathbf{V}(f, h, d)$, $\mathbf{V}=\left(\Phi_{1}, \Phi_{2}, \Phi_{3}\right)$, whose arguments are triples consisting of a meromorphic function $f$, a meromorphic vector field $h$, and a meromorphic differential 1-form $d$ on an open set $U \subset \mathbb{C}$ or, more general, on a Riemann surface $\Sigma$. Most of them are natural in the sense of 'natural operators' as considered in [8].

The special case $d(z)=0$ related to minimal curves in $\mathbb{C}^{3}$ and minimal surfaces in $\mathbb{R}^{3}$ is of main interest. We start with the invariant construction of a sequence $\mathbf{V}^{(n)}$ of natural operators assigning to each pair $(\mathbf{f}, \mathbf{h})$ consisting of a meromorphic function $\mathbf{f}$ and a meromorphic vector field $\mathbf{h}$ on $\Sigma$ a minimal curve $\mathbf{V}^{(n)}(\mathbf{f}, \mathbf{h}): \Sigma \rightarrow \mathbb{C}^{3}$. The operator $\mathbf{V}^{(3)}$ is bijective and equivariant on a generic set of pairs $(\mathbf{f}, \mathbf{h})$.

Algebraic representation formulas of minimal surfaces that arise from evolutes and caustics of curves in $\mathbb{R}^{2}$ in connection with the Björling representation formula are discussed.

We apply the computer algebra system Mathematica to handle big algebraic expressions describing these differential operators and to provide graphical examples of minimal surfaces produced by them.
\end{abstract}

1. Introduction. We define a minimal curve in $\mathbb{C}^{3}$ as a meromorphic mapping $\Phi$ : $\Sigma \rightarrow \mathbb{C}^{3}$ of a Riemann surface $\Sigma$ into $\mathbb{C}^{3}$ such that $\langle\mathbf{d} \Phi, \mathbf{d} \Phi\rangle=0$, where $\langle.,$.$\rangle is the$ bilinear extension of the ordinary Euclidean scalar product of $\mathbb{R}^{3}$ to $\mathbb{C}^{3}$. Let us denote by $\operatorname{Min}_{\Sigma}$ the set of all minimal curves defined on $\Sigma$. Two elements of Min $\Sigma$ that differ by a constant vector are considered as identical.

Minimal curves are in close relation to minimal surfaces in $\mathbb{R}^{3}$ : The set of real parts $M^{2}=\{\Re(\Phi(p)) \mid p \in \Sigma \backslash\{$ poles of $\Phi\}\} \subset \mathbb{R}^{3}$ is a minimal surface with isolated branch points and any local holomorphic chart $z$ of $\Sigma \backslash\{$ poles of $\Phi$ \} defines a conformal parametrization of the corresponding open part of $M^{2}$ (see [1], [3], [7], [9]).

2000 Mathematics Subject Classification: Primary 53A10; Secondary 53A05, 49Q05.

The paper is in final form and no version of it will be published elsewhere. 
Definition 1. Let $U \subset \mathbb{C}$ be an open subset and $\mathcal{A}_{U}$ the field of meromorphic functions on $U$. A local algebraic representation formula (ARF) in one function for minimal curves is a (nonlinear) differential operator $\mathcal{R}: \mathcal{A}_{U} \rightarrow \operatorname{Min}_{U}$. More precisely, $\mathcal{R}$ is an ARF if there exists a 3 -dimensional vector $\mathbf{V}=\mathbf{V}\left(x, y_{0}, y_{1}, \ldots, y_{m}\right)$ of meromorphic functions of $m+2$ variables such that $\mathcal{R}(f)(z)=\mathbf{V}\left(z, f(z), f^{\prime}(z), f^{\prime \prime}(z), \ldots, f^{(m)}(z)\right)$ for all $z \in U$, where $m$ is the order of $\mathcal{R}$. Algebraic representation formulas on $U$ in $k$ functions, $k \geq 1$, are defined as differential operators $\mathcal{R}: \underbrace{\mathcal{A}_{U} \times \cdots \times \mathcal{A}_{U}}_{k} \rightarrow \operatorname{Min}_{U}$ in an analogous way.

We mention two simple examples of algebraic representation formulas: The Study formula $\mathcal{R}_{1}(f)(z)$ is obtained from the Weierstraß formula (2.2) by replacing the argument function $d$ by $f^{\prime \prime \prime}$. Another one, denoted by $\mathcal{R}_{2}(f)(z)$, is an adaptation of an algebraic solution of the differential equation $\left(\left(y^{\prime}(x)\right)^{2}+\left(z^{\prime}(x)\right)^{2}=1\right.$ found in [2] (page 53). $\mathcal{R}_{2}$ is established there with the help of normal forms of Pfaffian systems. It appears also as a special case of a deformation operator $\Delta_{\Phi, h}$ of minimal curves $\Phi$, defined below in section 4. Substituting in (4.3) for $\Phi$ the minimal curve $\Phi(z)=(\cos z, \sin z,-i z)$ of the catenoid yields the formula $\mathcal{R}_{2}$.

$$
\begin{aligned}
& \mathcal{R}_{1}(f)(z)=\left(\begin{array}{c}
-2 f(z)+2 z f^{\prime}(z)-\left(z^{2}-1\right) f^{\prime \prime}(z) \\
i\left(2-2 z f^{\prime}(z)+\left(z^{2}+1\right) f^{\prime \prime}(z)\right) \\
2\left(z f^{\prime \prime}(z)-f^{\prime}(z)\right)
\end{array}\right) \\
& \mathcal{R}_{2}(h)(z)=\left(\begin{array}{c}
\sin (z) h^{\prime \prime}(z)-\cos (z) h^{\prime}(z) \\
\cos (z) h^{\prime \prime}(z)+\sin (z) h^{\prime}(z) \\
i\left(h(z)+h^{\prime \prime}(z)\right)
\end{array}\right) .
\end{aligned}
$$

We give in section 2 an invariant definition of a sequence $\mathbb{P}^{(n)}$ of operators that assign to each pair $(\mathbf{f}, \mathbf{h})$ consisting of a meromorphic function $\mathbf{f}$ and a vector field $\mathbf{h}$ on an arbitrary Riemann surface a meromorphic differential 1-forms $\omega$ such that $\mathbf{f}^{k} \omega$ is exact for $0 \leq k<n$. Combined with the Weierstraß formula they provide invariantly defined ARF's $\mathbf{V}^{(n)}$. In section 3 we look for algebraic generalizations of the sequence $\mathbb{P}^{(n)}$, replacing the field of meromorphic functions by an arbitrary commutative $\mathbb{C}$-algebra $\mathbf{A}$, the vector space of meromorphic differential 1-forms by a free $\mathbf{A}$-module of rank 1 and the complex exterior differential by a differentiation $\mathcal{D}: \mathbf{A} \rightarrow \mathbf{L}$. We derive under these general assumptions some properties of injectivity and equivariance.

In section 4 we show that $i \mathbf{V}^{(3)}$ agrees with an ARF discussed in two of our earlier papers. In section 5 we discuss an $\operatorname{ARF} \mathbf{V}(\mathbf{f}, \mathbf{h}, \omega)$ for meromorphic curves with arbitrary preset arc length differential $\omega(z) \neq 0$ and establish an algebraic bijection $\Omega$ between the invariants 'curvature' and 'torsion' of $\mathbf{V}(\mathbf{f}, \mathbf{h}, \omega)$ and the invariants of the minimal curves determined by $\mathbf{f}$ and $\mathbf{h}$. The operator $\mathbf{V}(\mathbf{f}, \mathbf{h}, \omega)$ gives rise to an $\mathrm{ARF}$ for meromorphic minimal curves in $\mathbb{C}^{4}$.

Section 6 is devoted to the fact that the arclength functions of the evolute and of certain caustics of a planar curve $\alpha(t)$ are algebraic expressions in $\alpha$, the curvature and the arc length element of $\alpha$. These expressions give ARF's in combination with the Björling representation formula.

For proofs we refer several times to our Mathematica packages. We end with some concrete graphical examples in section 7 . 
2. Natural representation formulas. Let $\Sigma$ be a connected Riemannian surface, $\mathcal{A}_{\Sigma}$ the field of meromorphic functions, $\mathcal{X}_{\Sigma}$ the space of meromorphic vector fields, $\mathcal{L}_{\Sigma}$ the space of meromorphic differential 1-forms on $\Sigma$ and

$$
\mathcal{M}_{\Sigma}(r, s, t)=\overbrace{\mathcal{A}_{\Sigma} \times \cdots \times \mathcal{A}_{\Sigma}}^{r} \times \overbrace{\mathcal{X}_{\Sigma} \times \cdots \times \mathcal{X}_{\Sigma}}^{s} \times \overbrace{\mathcal{L}_{\Sigma} \times \cdots \times \mathcal{L}_{\Sigma}}^{s} .
$$

Put $k=r+s+t$. In a local chart $\varphi: V \subset \Sigma: \rightarrow U \subset \mathbb{C}$ elements of $\mathcal{M}_{\Sigma}(r, s, t)$ are described as $k$-tuples $\left(f_{1}, \ldots, f_{r}, h_{1}, \ldots, h_{s}, d_{1}, \ldots, d_{t}\right)$ of functions on $U$ that transform under changes of coordinate systems in the usual way as functions, vector fields and differential forms respectively.

Definition 2. A local ARF $\mathcal{R}\left(g_{1}, \ldots, g_{k}\right)$ acting on $k$-tuples $\left(g_{1}, \ldots, g_{k}\right) \in \mathcal{A}_{\mathbb{C}} \times \cdots \times$ $\mathcal{A}_{\mathbb{C}}$ of meromorphic functions is called natural of type $(r, s, t)$ if for any Riemann surface $\Sigma$ there exists a differential operator $\mathcal{R}_{\Sigma}: \mathcal{M}_{\Sigma}(r, s, t) \rightarrow \operatorname{Min}_{\Sigma}$ such that for any local chart $\varphi: V \subset \Sigma \rightarrow U \subset \mathbb{C}$ and for any element $\left(\mathbf{f}_{1}, \ldots, \mathbf{f}_{r} \mathbf{h}_{1}, \ldots, \mathbf{h}_{s}, \omega_{1}, \ldots, \omega_{t}\right) \in \mathcal{M}_{\Sigma}(r, s, t)$

$$
\mathcal{R}_{\Sigma}\left(\mathbf{f}_{1}, \ldots, \mathbf{f}_{r} \mathbf{h}_{1}, \ldots, \mathbf{h}_{s}, \omega_{1}, \ldots, \omega_{t}\right) \circ \varphi^{-1}=\mathcal{R}\left(f_{1}, \ldots, f_{r}, h_{1}, \ldots, h_{s}, d_{1}, \ldots, d_{t}\right),
$$

where $\left(f_{1}, \ldots, f_{r}, h_{1}, \ldots, h_{s}, d_{1}, \ldots, d_{t}\right)$ are the local expressions of the global objects $\left(\mathbf{f}_{1}, \ldots, \mathbf{f}_{r}, \mathbf{h}_{1}, \ldots, \mathbf{h}_{s}, \omega_{1}, \ldots, \omega_{t}\right)$ with respect to $\varphi$.

A local $\operatorname{ARF} \mathcal{R}\left(g_{1}, \ldots, g_{k}\right)$ is natural of type $(r, s, t)$ (where $k=r+s+t$ ) if and only if for any holomorphic transformation $\tau: U_{1} \rightarrow U_{2}$ between open subsets $U_{1}, U_{2} \subset \mathbb{C}$ and for any $k$ meromorphic functions $f_{1}, \ldots, f_{r}, h_{1}, \ldots, h_{s}, d_{1}, \ldots, d_{t}$ one has

$$
\begin{aligned}
\mathcal{R}\left(f_{1}, \ldots, f_{r}, h_{1}, \ldots, h_{s}, d_{1}, \ldots, d_{t}\right) \circ \tau \\
\quad=\mathcal{R}\left(f_{1} \circ \tau, \ldots, f_{r} \circ \tau, \frac{h_{1} \circ \tau}{\tau^{\prime}}, \ldots, \frac{h_{s} \circ \tau}{\tau^{\prime}}, \tau^{\prime}\left(d_{1} \circ \tau\right), \ldots, \tau^{\prime}\left(d_{t} \circ \tau\right)\right) .
\end{aligned}
$$

We are going to construct an infinite sequence of natural ARF's by a direct procedure. Denote by $\mathcal{M}_{\Sigma}^{*}(1,0,1) \subset \mathcal{M}_{\Sigma}(1,0,1)$ the subset of pairs $(\mathbf{f}, \omega) \in \mathcal{A}_{\Sigma} \times \mathcal{L}_{\Sigma}$ such that $\omega$, $\mathbf{f} \omega$ and $\mathbf{f}^{2} \omega$ are exact, i.e., we demand that there exist functions $\varphi_{0}, \varphi_{1}, \varphi_{2} \in \mathcal{A}_{\Sigma}$ with $\mathbf{d} \varphi_{i}=\mathbf{f}^{i} \omega$ for $i=0,1,2$. The Weierstraß formula

$$
\mathbf{W}(f, d)(z)=\int_{z_{0}}^{z} \frac{d(\zeta)}{2}\left(\begin{array}{c}
1-f^{2}(\zeta) \\
i\left(1+f^{2}(\zeta)\right) \\
2 f(\zeta)
\end{array}\right) \mathbf{d} \zeta
$$

satisfies $\mathbf{W}\left(f \circ \tau, \tau^{\prime}(d \circ \tau)\right)=\mathbf{W}(f, d) \circ \tau$. This is a special case of $(2.1)$. We therefore consider $\mathbf{W}$ as a (nonalgebraic) natural representation formula $\mathbf{W}: \mathcal{M}_{\Sigma}^{*}(1,0,1) \rightarrow \operatorname{Min}_{\Sigma}$ of type $(1,0,1)$.

Now we define a sequence $\mathbb{P}^{(n)}: \mathcal{M}_{\Sigma}(1,1,0) \rightarrow \mathcal{L}_{\Sigma}=\mathcal{M}_{\Sigma}(0,0,1)$ of operators with the following property: Writing the first argument as index, we have $\left(\mathbf{f}, \mathbb{P}_{\mathbf{f}}^{(n)}(\mathbf{h})\right) \in \mathcal{M}_{\Sigma}^{*}(1,0,1)$ for all $(\mathbf{f}, \mathbf{h}) \in \mathcal{A}_{\Sigma} \times \mathcal{X}_{\Sigma}$ and $n \geq 3$. $\mathbb{P}^{(n)}$ is given recursively by

$$
\mathbb{P}_{\mathbf{f}}^{(0)}(\mathbf{h})=[\mathbf{d f}, \mathbf{h}] \mathbf{d f} \quad \text { and } \quad \mathbb{P}_{\mathbf{f}}^{(n)}(\mathbf{h})=\mathbf{d}\left(\frac{\mathbb{P}_{\mathbf{f}}^{(n-1)}(\mathbf{h})}{\mathbf{d f}}\right) .
$$

where $[.,$.$] denotes the pairing of differential 1-forms with vector fields and the quotient$ $\mathbb{P}_{\mathbf{f}}^{(n-1)}(\mathbf{h}) / \mathbf{d f}$ denotes the unique meromorphic function $\varphi$ such that $\mathbb{P}_{\mathbf{f}}^{(n-1)}(\mathbf{h})=\varphi \mathbf{d f}$. 
In a local chart $z: V \subset \Sigma \rightarrow U \subset \mathbb{C}$ on $\Sigma$, putting $f=z^{*} \mathbf{f}=\mathbf{f} \circ z^{-1}$ and $h=z_{*} \mathbf{h}$ the recursion formula $(2.3)$ becomes $\mathbb{P}_{f}^{(0)}(h)=f^{\prime 2} h, \mathbb{P}_{f}^{(1)}(h)=\left(f^{\prime} h\right)^{\prime}$ and $\mathbb{P}_{f}^{(n)}(h)=$ $\left(\mathbb{P}_{n-1}^{(n)}(f, h) / f^{\prime}\right)^{\prime}$. The local expressions of the first members of the sequence $\mathbb{P}_{f}^{(n)}(h)$ are

$$
\left\{\begin{aligned}
\mathbb{P}_{f}^{(1)}(h) & =f^{\prime} h^{\prime}+h f^{\prime \prime}, \quad \mathbb{P}_{f}^{(2)}(h)=\frac{f^{\prime} h^{\prime} f^{\prime \prime}-h f^{\prime \prime 2}+f^{\prime 2} h^{\prime \prime}+h f^{\prime} f^{(3)}}{f^{\prime 2}} \\
\mathbb{P}_{f}^{(3)}(h) & =\frac{-1}{f^{\prime 4}}\left(3 f^{\prime} h^{\prime} f^{\prime \prime 2}+3 h f^{\prime \prime 3}+2 f^{\prime 2} h^{\prime} f^{(3)}\right. \\
& \left.-4 h f^{\prime} f^{\prime \prime} f^{(3)}+f^{\prime 3} h^{(3)}+h f^{\prime 2} f^{(4)}\right) \\
\mathbb{P}_{f}^{(4)}(h) & =\frac{1}{f^{\prime 6}}\left(h f^{\prime 3} f^{(5)}+15 f^{\prime} h^{\prime} f^{\prime \prime 3}-15 h f^{\prime \prime 4}-3 f^{\prime 2} f^{\prime \prime 2} h^{\prime \prime}\right. \\
& +25 h f^{\prime} f^{\prime \prime 2} f^{(3)}+2 f^{\prime 3} h^{\prime \prime} f^{(3)}-4 h f^{\prime 2}\left(f^{(3)}\right)^{2}-2 f^{\prime 3} f^{\prime \prime} h^{(3)} \\
& \left.+3 f^{\prime 3} h^{\prime} f^{(4)}-7 h f^{\prime 2} f^{\prime \prime} f^{(4)}+f^{\prime 4} h^{(4)}-16 f^{\prime 2} h^{\prime} f^{\prime \prime} f^{(3)}\right)
\end{aligned}\right.
$$

Before going on with the construction, let us turn to the question of inverting $\mathbb{P}^{(n)}$. Obviously, each $\mathbb{P}_{\mathbf{f}}^{(n)}(\mathbf{h})$ is $\mathbb{C}$-linear in $\mathbf{h}$ and the equation $\mathbb{P}_{\mathbf{f}}^{(n)}(\mathbf{h})=\omega$ is an ordinary linear differential equation of order $n$. It can be solved by inverting the recursion procedure defining $\mathbb{P}^{(n)}$.

Choose a fixed $\mathbf{f} \in \mathcal{A}_{\Sigma}$ and assume that we have assigned to the operator $\mathbf{d}: \mathcal{A}_{\Sigma} \rightarrow \mathcal{L}_{\Sigma}$ a right inverse $\omega \in \mathcal{L}_{\mathbf{f}} \rightarrow \int \omega \in \mathcal{A}_{\Sigma}$, defined on a linear subspace $\mathcal{L}_{\mathbf{f}} \subset \mathcal{L}_{\Sigma}$. By means of this integral a sequence $\mathbb{Q}_{f}^{(n)}$ of right inverse operators to $\mathbb{P}_{f}^{(n)}$ is defined recursively by $\mathbb{Q}_{f}^{(0)}(\omega)=\omega / f^{\prime 2}, \mathbb{Q}_{f}^{(n+1)}(\omega)=\mathbb{Q}_{f}^{(n)}\left(f^{\prime} \int \omega\right)$. These operators satisfy $\mathbb{P}_{f}^{(n)}\left(\mathbb{Q}_{f}^{(n)}(\omega)\right)=\omega$ for all exact forms $\omega \in \mathcal{L}_{\Sigma}$.

Proposition 2.1. $\mathbb{Q}_{f}^{(n)}$ can be computed with the following explicit formula:

$$
\mathbb{Q}_{f}^{(n+1)}(\omega)=\frac{1}{f^{\prime}}\left(\frac{1}{n !} \sum_{i=0}^{n}(-1)^{i}\left(\begin{array}{c}
n \\
i
\end{array}\right) f^{n-i} \int\left(f^{i} \omega\right)+\sum_{i=0}^{n-1} c_{i} f^{i}\right)
$$

where $c_{0}, \ldots, c_{n-1}$ are constants of integration.

Proof. We show that $(2.5)$ satisfies the condition $\mathbb{Q}_{f}^{(n+1)}(\omega)=\mathbb{Q}_{f}^{(n)}\left(f^{\prime} \int \omega\right)$. Integration by parts gives

$$
\int\left(f^{i} f^{\prime} \int \omega\right)=\frac{1}{i+1}\left(f^{i+1} \int \omega-\int\left(f^{i+1} \omega\right)+\widetilde{c}_{n-i-1}\right), \quad i=0, \ldots, n-1,
$$

where $\widetilde{c}_{n-i-1} \in \mathbb{C}$ are constants of integration. Therefore

$$
\begin{aligned}
\mathbb{Q}_{f}^{(n)}\left(f^{\prime} \int \omega\right)= & \frac{1}{f^{\prime}}\left\{\frac{1}{(n-1) !} \sum_{i=0}^{n-1}(-1)^{i}\left(\begin{array}{c}
n-1 \\
i
\end{array}\right) f^{n-i-1} \int\left(f^{i} f^{\prime} \int \omega\right)+\sum_{i=0}^{n-1} c_{i} f^{i}\right\} \\
= & \frac{1}{f^{\prime}}\left\{\frac{1}{(n-1) !} \sum_{i=0}^{n-1}(-1)^{i}\left(\begin{array}{c}
n-1 \\
i
\end{array}\right) f^{n-i-1} \star\right. \\
& \left.\frac{1}{i+1}\left(f^{i+1} \int \omega-\int\left(f^{i+1} \omega\right)+\widetilde{c}_{n-i-1}\right)+\sum_{i=0}^{n-1} c_{i} f^{i}\right\} \\
= & A-B+C,
\end{aligned}
$$


with

$$
\begin{aligned}
& A=\frac{1}{f^{\prime}(n-1) !} \sum_{i=0}^{n-1}(-1)^{i} \frac{1}{i+1}\left(\begin{array}{c}
n-1 \\
i
\end{array}\right) f^{n} \int \omega \\
& B=\frac{1}{f^{\prime}(n-1) !} \sum_{i=0}^{n-1}(-1)^{i} \frac{1}{i+1}\left(\begin{array}{c}
n-1 \\
i
\end{array}\right) f^{n-i} \int f^{i+1} \omega, \\
& C=\frac{1}{f^{\prime}(n-1) !} \sum_{i=0}^{n-1}(-1)^{i} \frac{1}{i+1}\left(\begin{array}{c}
n-1 \\
i
\end{array}\right) \widetilde{c}_{n-i-1} f^{n-i-1}+c_{i} f^{i} .
\end{aligned}
$$

Finally, the equation $\frac{1}{i+1}\left(\begin{array}{c}n-1 \\ i\end{array}\right)=\frac{1}{n}\left(\begin{array}{c}n \\ i+1\end{array}\right)$ yields

$$
A=\frac{1}{f^{\prime}(n-1) !} \frac{1}{n} f^{n} \int \omega, \quad B=\frac{1}{f^{\prime}(n-1) !} \frac{1}{n} \sum_{i=1}^{n}(-1)^{i-1}\left(\begin{array}{c}
n \\
i
\end{array}\right) f^{n-i} \int\left(f^{i} \omega\right) .
$$

$C$ has again the form $C=\sum_{i=0}^{n} c_{i}^{*} f^{i}$ with some new constants $c_{i}^{*}$ resulting from $\widetilde{c}_{n-i-1}$ and $c_{i}$. Altogether $A-B+C=\mathbb{Q}_{f}^{(n+1)}(\omega)$.

REMARK 1. Equation (2.5) can also be applied to the case when $\Sigma$ is not simply connected and when the forms have poles. By proposition 2.2 below, if $\omega=\mathbb{P}_{\mathbf{f}}^{(n)}(\mathbf{h})$, then all forms $\mathbf{f}^{i} \omega, i=0, \ldots, n-1$, are exact. Therefore it is possible to choose $\mathcal{L}_{\mathbf{f}}$ as the image of $\mathbb{P}_{\mathbf{f}}^{(n)}$ and define $\int$ on $\mathcal{L}_{\mathbf{f}} \int: \mathcal{L}_{\mathbf{f}} \subset \mathcal{L} \rightarrow \mathcal{A}$ so that $\int \circ \mathbf{d}=\operatorname{Id}_{\mathbf{d}(\mathcal{A})}$.

We will show that for $n \geq 3$ the following special kind of composition of $\mathbb{P}^{(n)}$ with the Weierstraß formula

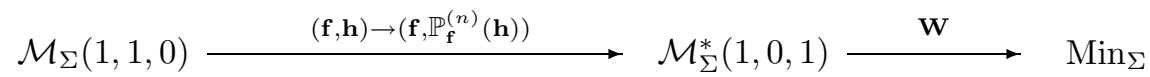

gives natural ARF's.

It is directly clear from the construction that each $\mathbb{P}^{(n)}$ maps $\mathcal{M}_{\Sigma}(1,1,0)$ to $\mathcal{L}_{\Sigma}$. We show that each pair $\left(\mathbf{f}, \mathbb{P}_{\mathbf{f}}^{(n)}(\mathbf{h})\right)$ belongs to $\mathcal{M}_{\Sigma}^{*}(1,0,1)$, i.e., that $\mathbf{f}^{0} \mathbb{P}_{\mathbf{f}}^{(n)}(\mathbf{h}), \mathbf{f}^{1} \mathbb{P}_{\mathbf{f}}^{(n)}(\mathbf{h})$ and $\mathbf{f}^{2} \mathbb{P}_{\mathbf{f}}^{(n)}(\mathbf{h})$ are exact forms. We have even more.

Proposition 2.2. The 1-forms $\mathbf{f}^{k} \mathbb{P}_{\mathbf{f}}^{(n)}(\mathbf{h})$ are exact for all $0 \leq k \leq n-1$ and all $(\mathbf{f}, \mathbf{h}) \in \mathcal{A}_{\Sigma} \times \mathcal{X}_{\Sigma}$.

Proof. We have to show that there exist functions $\varphi_{k, n}(\mathbf{f}, \mathbf{h}) \in \mathcal{A}_{\Sigma}$, depending on $(\mathbf{f}, \mathbf{h}) \in \mathcal{M}_{\Sigma}(1,1,0)$, with $\mathbf{d} \varphi_{k, n}(\mathbf{f}, \mathbf{h})=\mathbf{f}^{k} \mathbb{P}_{\mathbf{f}}^{(n)}(\mathbf{h})$. From $(2.3)$ we infer that $\mathbb{P}_{\mathbf{f}}^{(1)}(\mathbf{h})=$ $\mathbf{d}[\mathbf{d f}, \mathbf{h}]$ and, consequently, $\varphi_{0,1}(\mathbf{f}, \mathbf{h})=[\mathbf{d f}, \mathbf{h}]$. For $n>1$ the functions $\varphi_{0, n}(\mathbf{f}, \mathbf{h})=$ $\mathbb{P}_{\mathbf{f}}^{(n-1)}(\mathbf{h}) / \mathbf{d f}$ satisfy $\mathbf{d} \varphi_{0, n}(\mathbf{f}, \mathbf{h})=\mathbb{P}_{\mathbf{f}}^{(n)}(\mathbf{h})$. Moreover, the product rule gives

$$
\mathbf{f}^{k} \mathbb{P}_{\mathbf{f}}^{(n)}(\mathbf{h})=\mathbf{f}^{k} \mathbf{d}\left(\frac{\mathbb{P}_{\mathbf{f}}^{(n-1)}(\mathbf{h})}{\mathbf{d f}}\right)=\mathbf{d}\left(\mathbf{f}^{k} \frac{\mathbb{P}_{\mathbf{f}}^{(n-1)}(\mathbf{h})}{\mathbf{d f}}\right)-k \mathbf{f}^{k-1} \mathbb{P}_{\mathbf{f}}^{(n-1)}(\mathbf{h}) .
$$

This shows that the operators $\varphi_{k, n}: \mathcal{M}_{\Sigma}(1,1,0) \rightarrow \mathcal{A}_{\Sigma}$ defined for $0<k<n$ recursively by

$$
\varphi_{k, n}(\mathbf{f}, \mathbf{h})=\mathbf{f}^{k} \frac{\mathbb{P}_{\mathbf{f}}^{(n-1)}(\mathbf{h})}{\mathbf{d f}}-k \varphi_{k-1, n-1}(\mathbf{f}, \mathbf{h})
$$

have the desired property. 
We have in particular

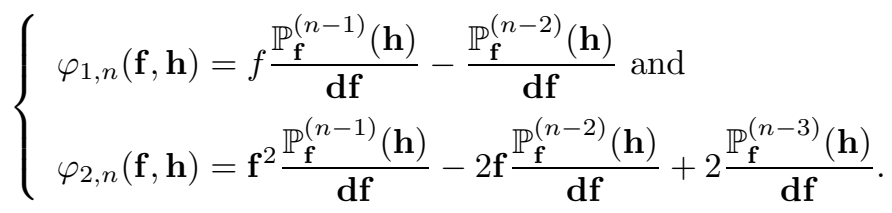

Local expressions of $\varphi_{k, 3}(f, h)$ for $k=0,1,2$ are

$$
\left\{\begin{aligned}
\varphi_{0,3}(f, h)= & \frac{1}{f^{\prime}(z)^{3}}\left(f^{\prime} h^{\prime} f^{\prime \prime}-h f^{\prime \prime 2}+f^{\prime 2} h^{\prime \prime}+h f^{\prime} f^{(3)}\right), \\
\varphi_{1,3}(f, h)= & \frac{1}{f^{\prime}(z)^{3}}\left(f f^{\prime 2} h^{\prime \prime}+f h f^{\prime} f^{(3)}-f^{\prime 3} h^{\prime}\right. \\
& \left.-h f^{\prime 2} f^{\prime \prime}+f f^{\prime} h^{\prime} f^{\prime \prime}-f h f^{\prime \prime 2}\right), \\
\varphi_{2,3}(f, h)= & \frac{1}{f^{\prime}(z)^{3}}\left(2 h f^{\prime 4}-2 f f^{\prime 3} h^{\prime}-2 f h f^{\prime 2} f^{\prime \prime}\right. \\
& \left.+f^{2} f^{\prime} h^{\prime} f^{\prime \prime}-f^{2} h f^{\prime \prime 2}+f^{2} f^{\prime 2} h^{\prime \prime}+f^{2} h f^{\prime} f^{(3)}\right) .
\end{aligned}\right.
$$

Observe that $\varphi_{k, n}(f, h)$ is $\mathbb{C}$-linear in $h$ for all $f$ and all $k<n$.

Theorem 2.1. Define $\mathbf{V}^{(n)}: \mathcal{M}_{\Sigma}(1,1,0) \rightarrow \operatorname{Min}_{\Sigma} b y$

$$
\mathbf{V}^{(n)}(\mathbf{f}, \mathbf{h})=\mathbf{W}\left(\mathbf{f}, \mathbb{P}_{\mathbf{f}}^{(n)}(\mathbf{h})\right) \text {. }
$$

For $n \geq 3$ the operators $\mathbf{V}^{(n)}$ are natural ARF's of type $(1,1,0)$.

The proof follows directly from the construction. The explicit form of $\mathbf{V}^{(n)}$ is

$$
\mathbf{V}^{(n)}(\mathbf{f}, \mathbf{h})(z)=\frac{1}{2}\left(\begin{array}{c}
\varphi_{0, n}(\mathbf{f}, \mathbf{h})(z)-\varphi_{2, n}(\mathbf{f}, \mathbf{h})(z) \\
i\left(\varphi_{0, n}(\mathbf{f}, \mathbf{h})(z)+\varphi_{2, n}(\mathbf{f}, \mathbf{h})(z)\right) \\
2 \varphi_{1, n}(\mathbf{f}, \mathbf{h})(z)
\end{array}\right)
$$

The operators $\mathbf{V}^{(n)}$ can be expressed in terms of $\mathbb{P}^{(n-1)}, \mathbb{P}^{(n-2)}, \mathbb{P}^{(n-3)}$ by means of (2.8):

$$
\frac{1}{2 \mathbf{d f}}\left(\begin{array}{c}
\left(1-\mathbf{f}^{2}\right) \mathbb{P}_{\mathbf{f}}^{(n-1)}(\mathbf{h})+2 \mathbf{f} \mathbb{P}_{\mathbf{f}}^{(n-2)}(\mathbf{h})-2 \mathbb{P}_{\mathbf{f}}^{(n-3)}(\mathbf{h}) \\
i\left(\left(1+\mathbf{f}^{2}\right) \mathbb{P}_{\mathbf{f}}^{(n-1)}(\mathbf{h})-2 \mathbf{f} \mathbb{P}_{\mathbf{f}}^{(n-2)}(\mathbf{h})+2 \mathbb{P}_{\mathbf{f}}^{(n-3)}(\mathbf{h})\right) \\
2\left(\mathbf{f} \mathbb{P}_{\mathbf{f}}^{(n-1)}(\mathbf{h})-\mathbb{P}_{\mathbf{f}}^{(n-2)}(\mathbf{h})\right)
\end{array}\right)
$$

$\mathbf{V}^{(3)}$ is of special importance. Since the Weierstraß formula (2.2) involves powers of $f$ up to order 2 it is by proposition 2.2 the first algebraic one among the operators $\mathbf{V}^{(n)}$. A second peculiarity is that $\mathbf{V}^{(3)}$ is equivariant and a bijective map from a generic subset $\mathcal{M}_{\Sigma}(1,1,0)$ onto the set of minimal curves $\Phi$ with $d \Phi_{1}-i d \Phi_{2} \neq 0$, as will be seen in sections 3 and 4 . Moreover, all $\mathbf{V}^{(n)}$ with $n \geq 3$ can be expressed by $\mathbf{V}^{(3)}$ :

Proposition 2.3. For each $n \geq 3$ the operator $\mathbf{V}^{(n)}$ satisfies the equation $\mathbf{V}^{(n)}(\mathbf{f}, \mathbf{h})$ $=\mathbf{V}^{(3)}\left(\mathbf{f}, \mathbf{h}_{n}\right)$, where the sequence $\mathbf{h}_{n}$ of meromorphic vector fields is defined by $\mathbf{h}_{3}=\mathbf{h}$,

$$
\mathbf{h}_{n}=\left[\mathbf{d}\left[\mathbf{d} \mathbf{f}, \mathbf{h}_{n-1}\right], \partial \mathbf{f}\right] \partial \mathbf{f}
$$

and where $\partial \mathbf{f}$ denotes the dual vector field of $\mathbf{d f}$ defined by $[\partial \mathbf{f}, \mathbf{d f}]=1$. 
Proof. It is sufficient to prove in a local coordinate system that $\mathbb{P}_{f}^{(n)}(h)=\mathbb{P}_{f}^{(3)}\left(h_{n}\right)$ where $f, h, h_{n}$ are the local expressions of $\mathbf{f}, \mathbf{h}$ and $\mathbf{h}_{n}$ respectively. The local version of $(2.13)$ is $h_{n}=\left(f^{\prime} h_{n-1}\right)^{\prime} / f^{\prime 2}$. We have $\mathbb{P}_{f}^{(3)}(h)=\mathbb{P}_{f}^{(3)}\left(h_{3}\right)$ by assumption. Assume that $\mathbb{P}_{f}^{(n-1)}(h)=\mathbb{P}_{f}^{(3)}\left(h_{n-1}\right)$. Since

$$
\mathbb{P}_{f}^{(n)}(h)=\left(\frac{\mathbb{P}_{f}^{(n-1)}(h)}{f^{\prime}}\right)^{\prime}=\left(\frac{\mathbb{P}_{f}^{(3)}\left(h_{n-1}\right.}{f^{\prime}}\right)^{\prime},
$$

it is sufficient to show that the equation

$$
\left(\frac{\mathbb{P}_{f}^{(3)}\left(h_{n-1}\right)}{f^{\prime}}\right)^{\prime}=\mathbb{P}_{f}^{(3)}\left(\frac{\left(f^{\prime} h_{n-1}\right)^{\prime}}{f^{\prime 2}}\right)
$$

holds for arbitrary functions $f$ and $h_{n-1}$. This can be done with the explicit form of $\mathbb{P}^{(3)}$ given in (2.4) and will become difficult. We have instructed the Mathematica program with the commands

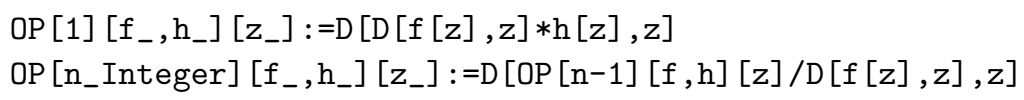

instead, encoding in this way the recursive definition $(2.3)$ of $\mathbb{P}_{f}^{(n)}(h)(z)$ as a Mathematica expression $\mathrm{OP}[\mathrm{n}][\mathrm{f}, \mathrm{h}][\mathrm{z}]$. Typing equation (2.15) as follows:

\section{$\mathrm{OP}[3][\mathrm{f}, \mathrm{D}[\mathrm{D}[\mathrm{f}[\#], \#] * \mathrm{~h}[\#], \#] / \mathrm{D}[\mathrm{f}[\#], \#]$ - $2 \&][\mathrm{z}]-$ \\ $\mathrm{D}[\mathrm{OP}[3][\mathrm{f}, \mathrm{h}][\mathrm{z}] / \mathrm{D}[\mathrm{f}[\mathrm{z}], \mathrm{z}], \mathrm{z}] / /$ Expand}

one obtains zero.

3. Algebraic generalizations, injectivity and equivariance of $\mathbf{V}^{(n)}$. We are going to study properties of invertibility and equivariance of the operator $\mathbf{V}^{(n)}$. In view of the algebraic character of the constructions of section 2 further considerations are performed under more general assumptions. Let us replace $\mathcal{A}_{\Sigma}, \mathcal{L}_{\Sigma}$ and $\mathcal{X}_{\Sigma}$ by an arbitrary commutative $\mathbb{C}$-algebra $\mathbf{A}$, a free $\mathbf{A}$-module $\mathbf{L}$ of rank 1 and the dual $\mathbf{L}^{*}$ of $\mathbf{L}$ respectively. The operator $\mathbf{d}$ of exterior differentiation is replaced by a 1-st order differential operator $\mathcal{D}: \mathbf{A} \rightarrow \mathbf{L}$, i.e., a $\mathbb{C}$-linear map such that $\mathcal{D}\left(\mathrm{f}_{1} \mathrm{f}_{2}\right)=\mathrm{f}_{2} \mathcal{D}\left(\mathrm{f}_{1}\right)+\mathrm{f}_{1} \mathcal{D}\left(\mathrm{f}_{2}\right)$ for all $\mathrm{f}_{1}, \mathrm{f}_{2} \in \mathbf{A}$. We assume that $\mathcal{D} \neq 0$.

$\mathbf{L}^{*}$ consists of all A-linear mappings of $\mathbf{L}$ into $\mathbf{A}$. Since we assume, that $\mathbf{A}$ acts freely and $\mathbf{L}$ is of rank 1 , for any two elements $\omega_{1}, \omega_{2} \in \mathbf{L}, \omega_{2} \neq 0$, there exists a unique element $Q\left(\omega_{1}, \omega_{2}\right) \in \mathbf{A}$ such that $Q\left(\omega_{1}, \omega_{2}\right) \omega_{2}=\omega_{1}$. As a map $Q: \mathbf{L} \times(\mathbf{L} \backslash\{0\}) \rightarrow \mathbf{A}, Q$ is A-linear in its first argument. We denote $Q\left(\omega_{1}, \omega_{2}\right)$ by $\omega_{1} / \omega_{2}$. For $\omega \in \mathbf{L}, \omega \neq 0$, we will denote by $\omega^{*} \in \mathbf{L}^{*}$ the A-linear mapping defined by $\left[\omega^{*}, \omega\right]=1$, where [,] denotes the dual pairing between $\mathbf{L}^{*}$ and $\mathbf{L}$. Then we have $\left[\omega^{*}, \omega_{1}\right]=\omega_{1} / \omega$ for any $\omega_{1} \in \mathbf{L}$.

Our aim is the construction of a sequence of algebraic solutions $\mathbf{V}^{(n)}: \mathbf{A} \backslash \operatorname{Ker}(\mathcal{D}) \rightarrow$ $\mathbf{A} \times \mathbf{A} \times \mathbf{A}$ of the following equation, generalizing the differential equation of minimal curves in $\mathbb{C}^{3}$ :

$$
\left(\mathcal{D} \circ \mathbf{V}_{1}^{(n)}\right)^{2}+\left(\mathcal{D} \circ \mathbf{V}_{2}^{(n)}\right)^{2}+\left(\mathcal{D} \circ \mathbf{V}_{3}^{(n)}\right)^{2}=0,
$$

where $\left(\mathcal{D} \circ \mathbf{V}_{i}^{(n)}(\mathrm{f})\right)^{2} \in \mathbf{L} \otimes_{\mathbf{A}} \mathbf{L}, \mathrm{f} \in \mathbf{A} \backslash \operatorname{Ker}(\mathcal{D})$ denotes the tensor product of $\mathcal{D} \circ \mathbf{V}_{i}^{(n)}(\mathrm{f})$ with itself and $\mathbf{V}^{(n)}=\left(\mathbf{V}_{1}^{(n)}, \mathbf{V}_{2}^{(n)}, \mathbf{V}_{3}^{(n)}\right)$. 
These assumptions on $\mathbf{A}, \mathbf{L}$ are rather strong and the only interesting model that we can provide is given by $d: \mathcal{A}_{\Sigma} \rightarrow \mathcal{L}_{\Sigma}$. We hope to weaken these assumptions in the future and that applications to other models become possible.

We define at first the sequence $\mathbf{P}^{(n)}: \mathbf{A} \backslash \operatorname{Ker}(\mathcal{D}) \rightarrow \mathbf{L}$ recursively by choosing $\mathbf{P}^{(0)}$ as an arbitrary map and putting

$$
\mathbf{P}^{(n)}=\mathcal{D}\left(\frac{\mathbf{P}^{(n-1)}(\mathrm{f})}{\mathcal{D}(\mathrm{f})}\right) \text { for } n \geq 1 .
$$

A double sequence $\theta_{k, n}$ of mappings $\theta_{k, n}: \mathbf{A} \backslash \operatorname{Ker}(\mathcal{D}) \rightarrow \mathbf{A}$ is defined for $n>0$, $0 \leq k<n$ and $\mathrm{f} \in \mathbf{A} \backslash \operatorname{Ker}(\mathcal{D})$ by

$$
\theta_{0, n}(\mathrm{f})=\frac{\mathbf{P}^{(n-1)}(\mathrm{f})}{\mathcal{D}(\mathrm{f})} \text { and } \theta_{k, n}(\mathrm{f})=\mathrm{f}^{k} \frac{\mathbf{P}^{(n-1)}(\mathrm{f})}{\mathcal{D}(\mathrm{f})}-k \theta_{k-1, n-1}(\mathrm{f}) .
$$

Then we obtain

Proposition 3.1. For $n>0$ and $0 \leq k<n$ we have

$$
\mathcal{D}\left(\theta_{k, n}(\mathrm{f})\right)=\mathrm{f}^{k} \mathbf{P}^{(n)}(\mathrm{f}) \text {. }
$$

Moreover, the operators $\mathbf{V}_{1}^{(n)}, \mathbf{V}_{2}^{(n)}, \mathbf{V}_{3}^{(n)}: \mathbf{A} \backslash \operatorname{Ker}(\mathcal{D}) \rightarrow \mathbf{A}$, given as components of the operator

$$
\mathbf{V}^{(n)}=\left(\begin{array}{c}
\mathbf{V}_{1}^{(n)}(\mathrm{f}) \\
\mathbf{V}_{2}^{(n)}(\mathrm{f}) \\
\mathbf{V}_{3}^{(n)}(\mathrm{f})
\end{array}\right)=\frac{1}{2}\left(\begin{array}{c}
\theta_{0, n}(\mathrm{f})-\theta_{2, n}(\mathrm{f}) \\
i\left(\theta_{0, n}(\mathrm{f})+\theta_{2, n}(\mathrm{f})\right) \\
2 \theta_{1, n}(\mathrm{f})
\end{array}\right)
$$

satisfy for $n \geq 3$ the condition (3.1).

Proof. The relation (3.4) is proved by induction. Further we obtain from (3.4)

$$
\mathcal{D}\left(\mathbf{V}^{(n)}(\mathrm{f})\right)=\frac{1}{2}\left(\begin{array}{c}
\mathbf{P}^{(n)}(\mathrm{f})-\mathrm{f}^{2} \mathbf{P}^{(n)}(\mathrm{f}) \\
i\left(\mathbf{P}^{(n)}(\mathrm{f})+\mathrm{f}^{2} \mathbf{P}^{(n)}(\mathrm{f})\right) \\
2 f \mathbf{P}^{(n)}(\mathrm{f})
\end{array}\right)
$$

Therefore,

$$
\begin{gathered}
\left(\mathcal{D} \circ \mathbf{V}_{1}^{(n)}(\mathrm{f})\right)^{2}+\left(\mathcal{D} \circ \mathbf{V}_{2}^{(n)}(\mathrm{f})\right)^{2}+\left(\mathcal{D} \circ \mathbf{V}_{3}^{(n)}(\mathrm{f})\right)^{2}= \\
\left(\left(1-\mathrm{f}^{2}\right)^{2}-\left(1+\mathrm{f}^{2}\right)^{2}+4 \mathrm{f}^{2}\right)\left(\mathbf{P}^{(n)}(\mathrm{f})\right)^{2}=0
\end{gathered}
$$

for any $\mathrm{f} \in \mathbf{A} \backslash \operatorname{Ker}(\mathcal{D})$.

Assume that $\Phi=\left(\Phi_{1}, \Phi_{2}, \Phi_{3}\right) \in \mathbf{A}^{3}$ and that there exists an $\mathrm{f} \in \mathbf{A} \backslash \operatorname{Ker}(\mathcal{D})$ with $\mathbf{V}^{(n)}(\mathrm{f})=\Phi$. Equation (3.6) implies

$$
\mathbf{P}^{(n)}(\mathrm{f})=\mathcal{D}\left(\Phi_{1}\right)-i \mathcal{D}\left(\Phi_{2}\right) \text { and } \mathrm{f}=\frac{\mathcal{D}\left(\Phi_{3}\right)}{\mathbf{P}^{(n)}(\mathrm{f})}=\frac{\mathcal{D}\left(\Phi_{3}\right)}{\mathcal{D}\left(\Phi_{1}\right)-i \mathcal{D}\left(\Phi_{2}\right)} .
$$

Consequently:

Proposition 3.2. The mapping

$$
\mathbf{V}^{(n) *}:\left(\Phi_{1}, \Phi_{2}, \Phi_{3}\right) \rightarrow \mathrm{f}=\frac{\mathcal{D}\left(\Phi_{3}\right)}{\mathcal{D}\left(\Phi_{1}\right)-i \mathcal{D}\left(\Phi_{2}\right)},
$$


defined on the subset of $\widehat{\mathbf{A}^{3}} \subset \mathbf{A}^{3}$ consisting of all triples $\left(\Phi_{1}, \Phi_{2}, \Phi_{3}\right)$ satisfying the inequality $\mathcal{D}\left(\Phi_{1}\right)-i \mathcal{D}\left(\Phi_{2}\right) \neq 0$, is a left inverse of $\mathbf{V}^{(n)}$, i.e., we have $\mathbf{V}^{(n) *} \circ \mathbf{V}^{(n)}=\mathrm{id} \mathbf{A}$. Therefore, $\mathbf{V}^{(n)}$ is a injective mapping of $\mathbf{A} \backslash \operatorname{Ker}\left(\mathbf{P}^{(n)}\right)$ into $\widehat{\mathbf{A}^{3}}$.

In addition to this, $\mathbf{P}^{(n-3)}$ can be reconstructed from $\mathbf{V}^{(n)}(\mathrm{f})$ in the following way:

Proposition 3.3. Define in analogy to (4.5) an algebraic version $\mathcal{W}(\mathrm{f}) \in\left(\mathbf{L}^{*}\right)^{3}$ of the Weierstraß formula by

$$
\mathcal{W}(\mathrm{f})=\frac{1}{2}\left(\begin{array}{c}
i\left(1-\mathrm{f}^{2}\right) \\
-1-\mathrm{f}^{2} \\
2 i \mathrm{f}
\end{array}\right) \mathcal{D}(\mathrm{f})^{*} \text { for } \mathrm{f} \in \mathbf{A} \backslash \operatorname{Ker}(\mathcal{D})
$$

For any $\mathrm{f} \in \mathbf{A} \backslash \operatorname{Ker}(\mathcal{D})$ and any $n \geq 3$ we have

$$
\left\langle\mathbf{V}^{(n)}(\mathrm{f}), \mathcal{W}(\mathrm{f})\right\rangle=\frac{-i \mathbf{P}^{(n-3)}(\mathrm{f})}{\mathcal{D}(\mathrm{f})} \mathcal{D}(\mathrm{f})^{*},
$$

where $\langle.,\rangle:. \mathbf{A}^{3} \times\left(\mathbf{L}^{*}\right)^{3} \rightarrow \mathbf{L}^{*}$ denotes the bilinear map given by

$$
\left\langle\left(\mathrm{f}_{1}, \mathrm{f}_{2}, \mathrm{f}_{3}\right),\left(\omega_{1}, \omega_{2}, \omega_{3}\right)\right\rangle=\mathrm{f}_{1} \omega_{1}+\mathrm{f}_{2} \omega_{2}+\mathrm{f}_{3} \omega_{3} .
$$

Proof. We derive from (3.3) the following analogue of (2.8):

$$
\left\{\begin{array}{l}
\theta_{1, n}(\mathrm{f})=f \frac{\mathbf{P}^{(n-1)}(\mathrm{f})}{\mathcal{D}(\mathrm{f})}-\frac{\mathbf{P}^{(n-2)}(\mathrm{f})}{\mathcal{D}(\mathrm{f})} \text { and } \\
\theta_{2, n}(\mathrm{f})=\mathrm{f}^{2} \frac{\mathbf{P}^{(n-1)}(\mathrm{f})}{\mathcal{D}(\mathrm{f})}-2 \mathrm{f} \frac{\mathbf{P}^{(n-2)}(\mathrm{f})}{\mathcal{D}(\mathrm{f})}+2 \frac{\mathbf{P}^{(n-3)}(\mathrm{f})}{\mathcal{D}(\mathrm{f})} .
\end{array}\right.
$$

Substituting these expressions in (3.5) yields the following analogue of (2.12):

$$
\mathbf{V}^{(n)}=\frac{1}{i \mathcal{D}(\mathrm{f})}\left(\mathbf{P}^{(n-1)}(\mathrm{f}) \mathbf{F}_{1}+\mathbf{P}^{(n-2)}(\mathrm{f}) \mathbf{F}_{2}+\mathbf{P}^{(n-3)}(\mathrm{f}) \mathbf{F}_{3}\right)
$$

where

$$
\mathbf{F}_{1}=\frac{1}{2}\left(\begin{array}{c}
i\left(1-\mathrm{f}^{2}\right) \\
-1-\mathrm{f}^{2} \\
2 i f
\end{array}\right), \quad \mathbf{F}_{2}=\left(\begin{array}{c}
i \mathrm{f} \\
\mathrm{f} \\
-i
\end{array}\right) \text { and } \mathbf{F}_{3}=\left(\begin{array}{c}
-i \\
-1 \\
0
\end{array}\right) .
$$

But $\left\langle\mathbf{F}_{1}, \mathcal{W}(\mathrm{f})\right\rangle=\left\langle\mathbf{F}_{2}, \mathcal{W}(\mathrm{f})\right\rangle=0$ and $\left\langle\mathbf{F}_{3}, \mathcal{W}(\mathrm{f})\right\rangle=\mathcal{D}(\mathrm{f})^{*}$. Therefore, equation (3.9) follows.

To take into account the dependence of $\mathbf{P}^{(n)}(\mathrm{f})$ on $\mathbf{P}^{(0)}(\mathrm{f})$ we put

$$
\mathbf{P}^{(0)}(\mathrm{f})=[\mathrm{h}, \mathcal{D}(\mathrm{f})] \mathcal{D}(\mathrm{f}) \text { for some element } \mathrm{h} \in \mathbf{L}^{*} .
$$

Then $\mathbf{P}^{(n)}$ becomes an operator that depends on a second argument $\mathrm{h} \in \mathbf{L}^{*}$. We write $\mathbf{P}^{(n)}(\mathrm{f}, \mathrm{h})$ to indicate this. Similarly $\mathbf{V}^{(n)}$ and $\theta_{k, n}$ become operators $\mathbf{V}^{(n)}(\mathrm{f}, \mathrm{h})$ and $\theta_{k, n}(\mathrm{f}, \mathrm{h})$ acting from $(\mathbf{A} \backslash \operatorname{Ker}(\mathcal{D})) \times \mathbf{L}^{*}$ to $\mathbf{A}^{3} \cdot \mathbf{P}^{(n)}, \theta_{k, n}$ and $\mathbf{V}^{(n)}$ are linear in the second argument. As a corollary to proposition 3.3 we obtain the following assertion about surjectivity of $\mathbf{P}^{(3)}$ :

COROLlary 1. Under the above assumptions we have

$$
\left\langle\mathbf{V}^{(3)}(\mathrm{f}, \mathrm{h}), \mathcal{W}(\mathrm{f})\right\rangle=-i \mathrm{~h} .
$$


Moreover, for fixed $\mathrm{f} \in \mathbf{A} \backslash \operatorname{Ker}(\mathcal{D})$ the kernel of the linear mapping

$$
\mathcal{D} \circ \mathbf{V}_{\mathrm{f}}^{(3)}: \mathrm{h} \in \mathbf{L}^{*} \rightarrow \mathcal{D} \circ \mathbf{V}^{(3)}(\mathrm{f}, \mathrm{h}) \in \mathbf{A}^{3}
$$

is the $\mathbb{C}$-vector subspace $\operatorname{Ker}\left(\mathcal{D} \circ \mathbf{V}_{\mathrm{f}}^{(3)}\right)=\left\{\left(\mathrm{c}_{0}+\mathrm{c}_{1} \mathrm{f}+\mathrm{c}_{2} \mathrm{f}^{2}\right) \mathcal{D}(\mathrm{f})^{*} ; \mathrm{c}_{0}, \mathrm{c}_{1}, \mathrm{c}_{2} \in \operatorname{Ker}(\mathcal{D})\right\}$ of $\mathbf{L}^{*}$. We denote the kernel of $\mathcal{D} \circ \mathbf{V}_{\mathrm{f}}^{(3)}$ by $\mathcal{K}_{\mathrm{f}}$.

The proof of the second assertion is based on (3.6) and similar to that of proposition 2.5.

Theorem 3.1. For each $\mathrm{f} \in \mathbf{A} \backslash \operatorname{Ker}(\mathcal{D})$ the operator $\mathbf{V}^{(3)}$ maps the kernel $\mathcal{K}_{\mathrm{f}}$ bijectively onto $\operatorname{Ker}(\mathcal{D})^{3} \subset \mathbf{A}^{3}$ via

$$
\mathbf{V}^{(3)}\left(\mathrm{f},\left(\mathrm{c}_{0}+\mathrm{c}_{1} \mathrm{f}+\mathrm{c}_{2} \mathrm{f}^{2}\right) \mathcal{D}(\mathrm{f})^{*}\right)=\left(\begin{array}{c}
\mathrm{c}_{0}+\mathrm{c}_{2} \\
-i \mathrm{c}_{0}+i \mathrm{c}_{2} \\
-\mathrm{c}_{1}
\end{array}\right) .
$$

Let $\mathcal{K}=\bigcup_{\mathrm{f} \in \mathbf{A}} \mathcal{K}_{\mathrm{f}} \subset \mathbf{A} \times \mathbf{L}^{*}$ be the union of all kernels $\mathcal{K}_{\mathrm{f}}$. $\mathbf{V}^{(3)}$ is a bijective map from $(\mathbf{A} \backslash \operatorname{Ker}(\mathcal{D})) \times \mathbf{L}^{*} \backslash \mathcal{K}$ onto the subset $\widehat{\mathbf{A}^{3}} \subset \mathbf{A}^{3}$ consisting of all triples $\left(\Phi_{1}, \Phi_{2}, \Phi_{3}\right)$ satisfying $\mathcal{D}\left(\Phi_{1}\right)-i \mathcal{D}\left(\Phi_{2}\right) \neq 0$.

Proof. The first assertion is obtained from (3.5) in the following way. Let $\mathrm{h}=$ $\left(\mathrm{c}_{0}+\mathrm{c}_{1} \mathrm{f}+\mathrm{c}_{2} \mathrm{f}^{2}\right) \mathcal{D}(\mathrm{f})^{*}$. We obtain $\mathbf{P}^{(0)}(\mathrm{f}, \mathrm{h})=\left(\mathrm{c}_{0}+\mathrm{c}_{1} \mathrm{f}+\mathrm{c}_{2} \mathrm{f}^{2}\right) \mathcal{D}(\mathrm{f}), \mathbf{P}^{(1)}(\mathrm{f}, \mathrm{h})=$ $\left(c_{0}+2 c_{2} f\right) \mathcal{D}(\mathrm{f})$ and $\mathbf{P}^{(2)}(\mathrm{f}, \mathrm{h})=2 \mathrm{c}_{2} \mathcal{D}(\mathrm{f})$. From (3.3) or (3.10) we infer $\theta_{0,3}=2 \mathrm{c}_{2}$, $\theta_{1,3}=-\mathrm{c}_{1}$ and $\theta_{2,3}=-2 \mathrm{c}_{0}$.

Next, let $\left(\mathrm{f}_{1}, \mathrm{~h}_{1}\right),\left(\mathrm{f}_{2}, \mathrm{~h}_{2}\right) \in(\mathbf{A} \backslash \operatorname{Ker}(\mathcal{D})) \times \mathbf{L}^{*} \backslash \mathcal{K}$ be given with $\mathbf{V}^{(3)}\left(\mathrm{f}_{1}, \mathrm{~h}_{1}\right)=$ $\mathbf{V}^{(3)}\left(\mathrm{f}_{2}, \mathrm{~h}_{2}\right)$. From proposition 3.2 we obtain $\mathrm{f}_{1}=\mathrm{f}_{2}$. Corollary 1 , equation (3.14), implies $\mathrm{h}_{1}=\mathrm{h}_{2}$. Therefore $\mathbf{V}^{(3)}$ is injective on $(\mathbf{A} \backslash \operatorname{Ker}(\mathcal{D})) \times \mathbf{L}^{*} \backslash \mathcal{K}$.

In order to prove surjectivity of $\mathbf{V}^{(3)}$, let $\Phi=\left(\Phi_{1}, \Phi_{2}, \Phi_{3}\right)^{\top} \in \widehat{\mathbf{A}^{3}}$ be given, define $\mathrm{f}$ and $\mathrm{h}$ by (3.7) and $\mathrm{h}=i\langle\Phi, \mathcal{W}(\mathrm{f})\rangle$ respectively. Let the vectors $\mathbf{F}_{1}, \mathbf{F}_{2}, \mathbf{F}_{3}$ be given as in (3.12) in terms of this $\mathrm{f}$. The determinant of the $3 \times 3$-matrix formed by the column vectors $\mathbf{F}_{1}, \mathbf{F}_{2}, \mathbf{F}_{3}$ is 1 . Therefore, there exist uniquely determined coefficients $a, b, c \in \mathbf{A}$ such that $\mathcal{D}(\Phi)=a \mathbf{F}_{1}+b \mathbf{F}_{2}+c \mathbf{F}_{3}$.

A direct computation with the defining equation (3.7) of $\mathrm{f}$ shows

$$
\left\langle\mathcal{D}(\Phi), \mathbf{F}_{1}\right\rangle=\left\langle\mathcal{D}(\Phi), \mathbf{F}_{2}\right\rangle=0 \text {, and }\left\langle\mathcal{D}(\Phi), \mathbf{F}_{3}\right\rangle=-i \mathcal{D}\left(\Phi_{1}\right)-\mathcal{D}\left(\Phi_{2}\right) .
$$

All scalar products $\left\langle\mathbf{F}_{i}, \mathbf{F}_{j}\right\rangle$ are zero, except for $\left\langle\mathbf{F}_{1}, \mathbf{F}_{3}\right\rangle=-\left\langle\mathbf{F}_{2}, \mathbf{F}_{2}\right\rangle=1$. Thus, looking at the scalar products of the equation $\mathcal{D}(\Phi)=a \mathbf{F}_{1}+b \mathbf{F}_{2}+c \mathbf{F}_{3}$ with $\mathbf{F}_{1}, \mathbf{F}_{2}, \mathbf{F}_{3}$ we find $a=-i \mathcal{D}\left(\Phi_{1}\right)-\mathcal{D}\left(\Phi_{2}\right)$ and $b=c=0$, i.e., $\mathcal{D}(\Phi)=-\left(i \mathcal{D}\left(\Phi_{1}\right)+\mathcal{D}\left(\Phi_{2}\right)\right) \mathbf{F}_{1}$.

On the other hand, $\mathcal{D}\left(\mathbf{V}^{(3)}(\mathrm{f}, \mathrm{h})\right)=-i \mathbf{P}^{(3)}(\mathrm{f}, \mathrm{h}) \mathbf{F}_{1}$ by (3.6). Therefore, it is sufficient to prove that $\mathbf{P}^{(3)}(\mathrm{f}, \mathrm{h})=\mathcal{D}\left(\Phi_{1}\right)-i \mathcal{D}\left(\Phi_{2}\right)$ to obtain $\mathcal{D}(\Phi)=\mathcal{D}\left(\mathbf{V}^{(3)}(\mathrm{f}, \mathrm{h})\right)$. We compute $\mathbf{P}^{(i)}(\mathrm{f}, \mathrm{h})$ for $i=0,1,2,3$ recursively with (3.2). Observe that $\mathcal{W}(\mathrm{f})=\mathbf{F}_{1} \mathcal{D}(\mathrm{f})^{*}, \mathcal{D}\left(\mathbf{F}_{1}\right)=$ $-\mathcal{D}(\mathrm{f}) \mathbf{F}_{2}$ and $\mathcal{D}\left(\mathbf{F}_{2}\right)=-\mathcal{D}(\mathrm{f}) \mathbf{F}_{3}$.

$$
\begin{aligned}
\mathbf{P}^{(0)}(\mathrm{f}, \mathrm{h}) & =i[\mathrm{~h}, \mathcal{D}(\mathrm{f})] \mathcal{D}(\mathrm{f})=i[\langle\Phi, \mathcal{W}(\mathrm{f})\rangle, \mathcal{D}(\mathrm{f})] \mathcal{D}(\mathrm{f}) \\
& =i\left[\left\langle\Phi, \mathbf{F}_{1}\right\rangle \mathcal{D}(\mathrm{f})^{*}, \mathcal{D}(\mathrm{f})\right] \mathcal{D}(\mathrm{f})=i\left\langle\Phi, \mathbf{F}_{1}\right\rangle \mathcal{D}(\mathrm{f}),
\end{aligned}
$$




$$
\begin{aligned}
& \mathbf{P}^{(1)}(\mathrm{f}, \mathrm{h})=\mathcal{D}\left(\frac{\mathbf{P}^{(0)}(\mathrm{f}, \mathrm{h})}{\mathcal{D}(\mathrm{f})}\right)=i \mathcal{D}\left(\left\langle\Phi, \mathbf{F}_{1}\right\rangle\right)=i\left\langle\mathcal{D}(\Phi), \mathbf{F}_{1}\right\rangle+i\left\langle\Phi, \mathcal{D}\left(\mathbf{F}_{1}\right)\right\rangle \\
&=-i\left\langle\Phi, \mathcal{D}(\mathrm{f}) \mathbf{F}_{2}\right\rangle=-i\left\langle\Phi, \mathbf{F}_{2}\right\rangle \mathcal{D}(\mathrm{f}), \\
& \mathbf{P}^{(2)}(\mathrm{f}, \mathrm{h})=\mathcal{D}\left(\frac{\mathbf{P}^{(1)}(\mathrm{f}, \mathrm{h})}{\mathcal{D}(\mathrm{f})}\right)=-i \mathcal{D}\left(\left\langle\Phi, \mathbf{F}_{2}\right\rangle\right)=i\left\langle\Phi, \mathbf{F}_{3}\right\rangle \mathcal{D}(\mathrm{f}), \\
& \mathbf{P}^{(3)}(\mathrm{f}, \mathrm{h})=\mathcal{D}\left(\frac{\mathbf{P}^{(2)}(\mathrm{f}, \mathrm{h})}{\mathcal{D}(\mathrm{f})}\right)=i \mathcal{D}\left(\left\langle\Phi, \mathbf{F}_{3}\right\rangle\right)=\mathcal{D}\left(\Phi_{1}\right)-i \mathcal{D}\left(\Phi_{2}\right) .
\end{aligned}
$$

Therefore $\mathcal{D}(\Phi)=\mathcal{D}\left(\mathbf{V}^{(3)}(\mathrm{f}, \mathrm{h})\right)$, i.e. $\Phi-\mathbf{V}^{(3)}(\mathrm{f}, \mathrm{h})=\overrightarrow{\mathrm{c}}=\left(\mathrm{c}_{0}, \mathrm{c}_{1}, \mathrm{c}_{2}\right)^{\top}$ with $\mathrm{c}_{i} \in \operatorname{Ker}(\mathcal{D})$.

Finally, since $-i \mathrm{~h}=\langle\Phi, \mathcal{W}(\mathrm{f})\rangle=\left\langle\mathbf{V}^{(3)}(\mathrm{f}, \mathrm{h}), \mathcal{W}(\mathrm{f})\right\rangle$, we have $\langle\overrightarrow{\mathrm{c}}, \mathcal{W}(\mathrm{f})\rangle=0$. This implies $\left\langle\vec{c}, \mathbf{F}_{1}\right\rangle=0$. Applying the operator $\mathcal{D}$ to this equation and using $\mathcal{D}\left(\mathbf{F}_{1}\right)=-\mathcal{D}(\mathrm{f}) \mathbf{F}_{2}$, $\mathcal{D}\left(\mathbf{F}_{2}\right)=-\mathcal{D}(\mathrm{f}) \mathbf{F}_{3}$, we get $\left\langle\vec{c}, \mathbf{F}_{2}\right\rangle=\left\langle\overrightarrow{\mathrm{c}}, \mathbf{F}_{3}\right\rangle=0$. Therefore $\overrightarrow{\mathrm{c}}=0$ and $\Phi=\mathbf{V}^{(3)}(\mathrm{f}, \mathrm{h})$.

The following considerations are concerned with equivariance of $\mathbf{V}^{(3)}$. We assume that $\mathbf{A}$ is a field as in the case of $\mathcal{A}_{\Sigma}$. Then we have $\mathbb{C} \subset \mathbf{A}$. An action of the group $\operatorname{Sl}(2, \mathbb{C})$ on $\mathbf{A} \backslash \mathbb{C}$ is defined by

$$
(\mathrm{A}, \mathrm{f}) \in \mathrm{Sl}(2, \mathbb{C}) \times(\mathbf{A} \backslash \mathbb{C}) \rightarrow \frac{a \mathrm{f}+b}{c \mathrm{f}+d} \in \mathbf{A} \backslash \mathbb{C}, \quad \mathrm{A}=\left(\begin{array}{ll}
a & b \\
c & d
\end{array}\right) \in \operatorname{Sl}(2, \mathbb{C}) .
$$

On the other hand, the action of the complex conformal group $\mathrm{CO}(3, \mathbb{C})$ on $\mathbf{L}^{3}$ given by matrix multiplication leaves the equation (3.1) invariant, i.e., for $\omega=\left(\omega_{1}, \omega_{2}, \omega_{3}\right)^{\top} \in$ $\mathbf{L}^{3}, \mathrm{~B} \in \mathrm{O}(3, \mathbb{C}), \omega^{\sharp}=\mathrm{B} \omega$ the equation $\omega_{1} \otimes \omega_{1}+\omega_{2} \otimes \omega_{2}+\omega_{3} \otimes \omega_{3}=0$ implies $\omega_{1}^{\sharp} \otimes \omega_{1}^{\sharp}+\omega_{2}^{\sharp} \otimes \omega_{2}^{\sharp}+\omega_{3}^{\sharp} \otimes \omega_{3}^{\sharp}=0$.

Proposition 3.4. Assume that $\mathbf{P}^{(0)}$ is given by $\mathbf{P}^{(0)}(\mathrm{f})=[\mathrm{h}, \mathcal{D}(\mathrm{f})] \mathcal{D}(\mathrm{f})$, where $\mathrm{h} \in$ $\mathbf{L}^{*}$ is a fixed element. Denote by $\mu: G l(2, \mathbb{C}) \rightarrow O(3, \mathbb{C})$ the group homomorphism sending a matrix $\mathrm{A}=\left(\begin{array}{ll}a & b \\ c & d\end{array}\right) \in G l(2, \mathbb{C})$ to the matrix $\mathrm{B}=\mu(\mathrm{A}) \in C O(3, \mathbb{C})$ given by

$$
\mathrm{B}=\frac{1}{2(a d-b c)}\left(\begin{array}{ccc}
a^{2}-b^{2}-c^{2}+d^{2} & i\left(a^{2}+b^{2}-c^{2}-d^{2}\right) & 2 c d-2 a b \\
i\left(b^{2}-c^{2}+d^{2}-a^{2}\right) & a^{2}+b^{2}+c^{2}+d^{2} & 2 i(a b+c d) \\
2 b d-2 a c & -2 i(a c+b d) & 2(b c+a d)
\end{array}\right) .
$$

The operator $\mathbf{V}^{(n)}$ is equivariant with respect to $\mu$, i.e., for all $\mathrm{f} \in \mathbf{A} \backslash \operatorname{Ker}(\mathcal{D})$,

$$
\mathbf{V}^{(n)}\left(\frac{a \mathrm{f}+b}{c \mathrm{f}+d}\right)=\mu(\mathrm{A}) \mathbf{V}^{(n)}(\mathrm{f}), \quad \mathrm{A}=\left(\begin{array}{ll}
a & b \\
c & d
\end{array}\right) \in S l(2, \mathbb{C}) .
$$

Proof. It is sufficient to prove (3.19) for the transformations $\mathrm{f} \rightarrow 1 / \mathrm{f}$ and $\mathrm{f} \rightarrow a+b \mathrm{f}$, $a, b \in \mathbb{C}$. The transformation $\mathrm{f} \rightarrow 1 / \mathrm{f}$ corresponds to the matrix $\mathrm{A}\left(\begin{array}{ll}0 & 1 \\ 1 & 0\end{array}\right)$ and $\mu(\mathrm{A})$ is the diagonal matrix $B=\operatorname{diag}(1,-1,-1)$. From our assumption $\mathbf{P}^{(0)}(\mathrm{f}, \mathrm{h})=[\mathrm{h}, \mathcal{D}(\mathrm{f})] \mathcal{D}(\mathrm{f})$ and the recursive definition (3.2) we infer

$$
\begin{aligned}
& \mathbf{P}^{(0)}\left(\frac{1}{\mathrm{f}}, \mathrm{h}\right)=\frac{\mathbf{P}^{(0)}(\mathrm{f}, \mathrm{h})}{\mathrm{f}^{4}}, \quad \mathbf{P}^{(1)}\left(\frac{1}{\mathrm{f}}, \mathrm{h}\right)=\frac{2 \mathbf{P}^{(0)}(\mathrm{f}, \mathrm{h})}{\mathrm{f}^{3}}-\frac{\mathbf{P}^{(1)}(\mathrm{f}, \mathrm{h})}{\mathrm{f}^{2}} \text { and } \\
& \mathbf{P}^{(2)}\left(\frac{1}{\mathrm{f}}, \mathrm{h}\right)=\mathbf{P}^{(2)}(\mathrm{f}, \mathrm{h})-\frac{2 \mathbf{P}^{(1)}(\mathrm{f}, \mathrm{h})}{\mathrm{f}}+\frac{2 \mathbf{P}^{(0)}(\mathrm{f}, \mathrm{h})}{\mathrm{f}^{2}} .
\end{aligned}
$$


Computing $\mathbf{V}^{(3)}(1 / \mathrm{f}, \mathrm{h})$ with $(3.11)$ and replacing $\mathbf{P}^{(i)}(1 / \mathrm{f}, \mathrm{h})$ for $i=0,1,2$ by these terms one obtains $\mathrm{BV}^{(3)}(\mathrm{f}, \mathrm{h})$.

The verification of (3.19) for the transformation $\mathrm{f} \rightarrow a+b \mathrm{f}$ is similar.

4. A universal natural algebraic representation formula. We are going to discuss relations of the natural algebraic representation formula $\mathbf{V}^{(3)}(\mathbf{f}, \mathbf{h})$ to deformations of minimal curves and minimal surfaces considered in two earlier papers and in unpublished Mathematica programs that can be found in [4] and to our Mathematica packages min.m and $n$-dimwei.m to be found under the electronic web adress given in [4]. These relations reveal geometric properties of $\mathbf{V}^{(3)}(\mathbf{f}, \mathbf{h})$.

We confine our considerations to minimal curves $\Phi(z)$ in $\operatorname{Min}_{\mathbb{C}}$ and assume that $\Phi(z)$ is generally curved, i.e., that $\Phi^{\prime}, \Phi^{\prime \prime}, \Phi^{\prime \prime \prime}$ are linearly independent. Basic differential invariants of $\Phi(z)$ are the canonical parameter $p(z)$ and the minimal curvature $\kappa_{\Phi}(z)$. The latter is also called Study parameter. One can define them by

$$
p^{\prime}(z)=i \sqrt[4]{\left\langle\Phi^{\prime \prime}(z), \Phi^{\prime \prime}(z)\right\rangle} \text { and } \kappa_{\Phi}^{2}(p)=\left\langle\frac{\mathbf{d}^{3} \Phi(p)}{\mathbf{d} p^{3}}, \frac{\mathbf{d}^{3} \Phi(p)}{\mathbf{d} p^{3}}\right\rangle
$$

respectively (see for instance [1] or [10]).

Locally, $\Phi(z)$ can be reparametrized to $p$. In the sequel we will denote by $\Phi^{\prime}, \Phi^{\prime \prime}, \ldots$ the derivatives with respect to $p$. Then we have $\left\langle\Phi^{\prime \prime}, \Phi^{\prime \prime}\right\rangle=-1,\left\langle\Phi^{\prime \prime \prime}, \Phi^{\prime \prime \prime}\right\rangle=\kappa^{2}$. From these equations and the minimality condition $\left\langle\Phi^{\prime}, \Phi^{\prime}\right\rangle=0$, all scalar products $\left\langle\Phi^{(i)}, \Phi^{(j)}\right\rangle$ of higher derivatives of $\Phi$ can be computed by successive differentiation. The scalar products $\left\langle\Phi^{(i)}(p), \Phi^{(j)}(p)\right\rangle$ depend only on $\kappa^{2}(p)$ and its derivatives. The first $4 \times 4$ of them are arranged in the following matrix.

$$
\left(\left\langle\Phi^{(i)}, \Phi^{(j)}\right\rangle\right)_{i, j=1}^{4}=\left(\begin{array}{cccc}
0 & 0 & 1 & 0 \\
0 & -1 & 0 & -\kappa^{2}(z) \\
1 & 0 & \kappa^{2}(z) & \kappa(z) \kappa^{\prime}(z) \\
0 & -\kappa^{2}(z) & \kappa(z) \kappa^{\prime}(z) & -\kappa^{4}(z)
\end{array}\right) .
$$

Proposition 4.1. For any minimal curve $\Phi$ the equation $\Phi^{(4)}=\kappa \kappa^{\prime} \Phi^{\prime}+\kappa^{2} \Phi^{\prime \prime}$ holds and for any meromorphic function $h$ the new curves

$$
\Delta_{\Phi, h}=\left(-h \kappa_{\Phi}^{2}+h^{\prime \prime}\right) \Phi^{\prime}-h^{\prime} \Phi^{\prime \prime}+h \Phi^{(3)} \text { and } \Psi_{\Phi, h}=\Phi+\Delta_{\Phi, h}
$$

are again minimal curves. Moreover, the tangent vector $\Delta_{\Phi, h}^{\prime}$ is parallel to $\Phi^{\prime}$, namely

$$
\Delta_{\Phi, h}^{\prime}=\left(-h^{\prime} \kappa_{\Phi}^{2}-h \kappa_{\Phi} \kappa_{\Phi}^{\prime}+h^{\prime \prime \prime}\right) \Phi^{\prime} .
$$

Proof. Starting with an ansatz $\Phi^{(4)}=a \Phi^{\prime}+b \Phi^{\prime \prime}+c \Phi^{\prime \prime \prime}$, the coefficients $a, b, c$ are obtained by computing the scalar products $\left\langle\Phi^{(4)}, \Phi^{(i)}\right\rangle, i=1,2,3$, with the appropriate entries of the matrix (4.2). In the same way, the equations $\left\langle\Delta_{\Phi, h}, \Delta_{\Phi, h}\right\rangle=0$ and $\left\langle\Psi_{\Phi, h}, \Psi_{\Phi, h}\right\rangle=0$ are verified.

We call $\Delta_{\Phi, h}$ the variation and $\Psi_{\Phi, h}$ the deformation of $\Phi$ by means of $h$. Since the variation involves only derivatives of $\Phi$, one can assume that $\Phi$ is given by an arbitrary representation formula that may contain integrations (for instance the Björling 
formulas (6.1) or (6.2)) to produce algebraic formulas. Let $\Phi(z)$ be the minimal curve $\Phi(z)=(\cos z, \sin z, i z)$ whose associated minimal surface is the catenoid. A direct computation shows that up to signs, $\Delta_{\Phi, \sqrt{-i} h}$ agrees with the $\operatorname{ARF} \mathcal{R}_{2}(h)(z)$ on page 110.

Substituting $g(z)=i / f^{\prime}(z)$ in the Weierstraß formula (2.2), a non-natural integral representation formula $\mathbf{W}_{f}^{*}$ of Weierstraß type is obtained:

$$
\mathbf{W}_{f}^{*}(z)=\int_{z_{0}}^{z} \frac{i}{2 f^{\prime}(\zeta)}\left(1-f^{2}(\zeta),-\left(1+f^{2}(\zeta)\right), 2 f(\zeta)\right) \mathbf{d} \zeta
$$

The curve $\mathbf{W}_{f}^{*}(z)$ is parametrized by its canonical parameter and $\mathbf{W}^{*}$ is a bijection onto the set of all minimal curves $\left(\Phi_{1}, \Phi_{2}, \Phi_{3}\right)$ in canonical parametrization satisfying the inequality $\Phi_{1}^{\prime} \neq i \Phi_{2}^{\prime}$.

The Study parameter of $\mathbf{W}_{f}^{*}(z)$ is the Schwarzian derivative

$$
\kappa_{\mathbf{W}_{f}^{*}}^{2}(z)=\mathcal{S}_{f}(z)=\frac{3 f^{\prime \prime 2}(z)-2 f^{\prime}(z) f^{(3)}(z)}{f^{\prime 2}(z)} .
$$

TheOREM 4.1. (i) The differential operator $\mathbf{V}: \mathcal{A}_{\mathbb{C}} \times \mathcal{A}_{\mathbb{C}} \rightarrow \operatorname{Min}_{\mathbb{C}}$ given by $\mathbf{V}(f, h)=$ $\Delta_{\mathbf{W}_{f}^{*}, h}$ is a natural algebraic representation formula of type $(1,1,0) . \mathbf{V}$ is $\mathbb{C}$-linear in $h$. Moreover, $\mathbf{V}=i \mathbf{V}^{(3)}$, where $\mathbf{V}^{(n)}$ is the sequence of ARF's defined in proposition (2.1).

(ii) Let $\mathcal{K} \subset \mathcal{A}_{\mathbb{C}} \times \mathcal{A}_{\mathbb{C}}$ be the subset, consisting of all pairs $(f, h)$ such that $h=$ $\left(c_{0}+c_{1} f+c_{2} f^{2}\right) / f^{\prime}, c_{0}, c_{1}, c_{2} \in \mathbb{C} . \mathbf{V}$ is a bijection of the complement of $\mathcal{K}$ onto the subset $\operatorname{Min}_{\mathbb{C}}^{*} \subset \operatorname{Min}_{\mathbb{C}}$ of minimal curves $\Phi$ such that $\Phi_{1}^{\prime}-i \Phi_{2}^{\prime} \neq 0$.

(iii) $\mathbf{V}$ is equivariant with respect to a certain Lie group homomorphism $\tilde{\mu}$ of $\mathrm{Gl}(2, \mathbb{C})$ onto $\mathrm{CO}(3, \mathbb{C})$ extending the double covering (3.18) $\mu: \mathrm{Sl}(2, \mathbb{C}) \rightarrow \mathrm{SO}(3, \mathbb{C})$ (see (3.18)). Here it is assumed that a matrix $M \in \mathrm{Gl}(2, \mathbb{C})$ with entries $a_{i j}$ acts on $\mathcal{A}_{\mathbb{C}} \times \mathcal{A}_{\mathbb{C}}$ by assigning to $(f, h) \in \mathcal{A}_{\mathbb{C}} \times \mathcal{A}_{\mathbb{C}}$ the pair $\left(f^{*}, h^{*}\right)$ given by $f^{*}=\left(a_{11}+a_{12} f\right) /\left(a_{21}+a_{22} f\right)$ and $h^{*}=\operatorname{det}(\mathrm{M}) \mathrm{h}$. The complex conformal group $\mathrm{Gl}(2, \mathbb{C})$ acts on $\operatorname{Min}_{\mathbb{C}}$ in the usual way by matrix multiplication

Proof. Most of these assertions have been proved in our papers [5] and [6]. The equality between $\mathbf{V}$ and $i \mathbf{V}^{(3)}$ can be proved by direct evaluation of the operator OP [n] [f, h] [z] of section 2 and comparing it with the explicit form of $\mathbf{V}(f, h)$ given below.

We mention that the inverse operator $\mathbf{V}^{-1}: \operatorname{Min}_{\mathbb{C}}^{*} \rightarrow \mathcal{A}_{\mathbb{C}} \times \mathcal{A}_{\mathbb{C}}$ is also a differential operator. For $\Phi=\left(\Phi_{1}, \Phi_{2}, \Phi_{3}\right) \in \operatorname{Min}_{\mathbb{C}}^{*}$ it is given by $\mathbf{V}^{-1}(\Phi)=(f, h)$, where $f=$ $\Phi_{3}^{\prime} /\left(\Phi_{1}^{\prime}-i \Phi_{2}^{\prime}\right)$ and $h=\left\langle\Phi, \mathbf{W}_{f}^{\prime}(z)\right\rangle$. The explicit form of $\mathbf{V}(f, h)$ is

$$
\frac{i}{2 f^{\prime 3}}\left(\begin{array}{c}
f^{\prime}\left(2 f f^{2} h^{\prime}+h^{\prime} f^{\prime \prime}-f^{2} h^{\prime} f^{\prime \prime}+f^{\prime} h^{\prime \prime}-f^{2} f^{\prime} h^{\prime \prime}\right)+ \\
h\left(-2 f^{\prime}+2 f f^{\prime 2} f^{\prime \prime}-f^{\prime \prime 2}+f^{2} f^{\prime \prime 2}+f^{\prime} f^{\prime \prime \prime}-f^{2} f^{\prime} f^{\prime \prime \prime}\right) \\
i\left(f^{\prime}\left(-2 f f^{\prime 2} h^{\prime}+h^{\prime} f^{\prime \prime}+f^{2} h^{\prime} f^{\prime \prime}+f^{\prime} h^{\prime \prime}+f^{2} f^{\prime} h^{\prime \prime}\right)+\right. \\
\left.h\left(2 f^{\prime}-2 f f^{\prime 2} f^{\prime \prime}-f^{\prime \prime 2}-f^{2} f^{\prime \prime 2}+f^{\prime} f^{\prime \prime \prime}+f^{2} f^{\prime} f^{\prime \prime \prime}\right)\right) \\
2\left(f^{\prime 2}\left(f h^{\prime \prime}-h f^{\prime \prime}\right)+f f^{\prime}\left(h^{\prime} f^{\prime \prime}+h f^{\prime \prime \prime}\right)\right)-f^{\prime 3} h^{\prime}-f h f^{\prime \prime}
\end{array}\right) .
$$

5. Algebraic representation formulas for curves with arbitrary arc length function. The formula (4.3) can be generalized to a representation formula of meromor- 
phic curves with arbitrary infinitesimal arc length element $d(z)$. One simply appends the summand $d(z) \Phi^{\prime}(z)$ to $\Delta_{\Phi, h}$, i.e. putting

$$
\widetilde{\Delta}(\Phi, h, d)=\left(-h \kappa_{\Phi}^{2}+h^{\prime \prime}\right) \Phi^{\prime}-h^{\prime} \Phi^{\prime \prime}+h \Phi^{(3)}+d \Phi^{\prime}
$$

one obtains with the formula $\Phi^{(4)}=\kappa \kappa^{\prime} \Phi^{\prime}+\kappa^{2} \Phi^{\prime \prime}$ of proposition 4.1

$$
\frac{\mathbf{d} \widetilde{\Delta}(\Phi, h, d)}{\mathbf{d} z}=\left(-h^{\prime} \kappa_{\Phi}^{2}-h \kappa_{\Phi} \kappa_{\Phi}^{\prime}+h^{\prime \prime \prime}+d^{\prime}\right) \Phi^{\prime}+d \Phi^{\prime \prime}
$$

and the equations $\left\langle\Phi^{\prime}, \Phi^{\prime}\right\rangle=\left\langle\Phi^{\prime}, \Phi^{\prime \prime}\right\rangle=0$ and $\left\langle\Phi^{\prime \prime}, \Phi^{\prime \prime}\right\rangle=-1$ (compare (4.2)) give

$$
\left\langle\frac{\mathbf{d} \widetilde{\Delta}(\Phi, h, d)(z)}{\mathbf{d} z}, \frac{\mathbf{d} \widetilde{\Delta}(\Phi, h, d)(z)}{\mathbf{d} z}\right\rangle=-d^{2}(z) .
$$

Definition 3. We specialize $\widetilde{\Delta}(\Phi, h, d)$ to the case $\Phi=\mathbf{W}_{f}^{*}$ and define a differential operator $\mathbf{V}$ assigning a curve to three functions by $\mathbf{V}(f, h, d)=\widetilde{\Delta}\left(\mathbf{W}_{f}^{*}, h, d\right)$.

Considerations similar to those of proposition 4.1 show that $\mathbf{V}(f, h, d)$ is an algebraic representation formula for curves with arbitrary arc length element $\mathbf{d} s^{2}=-d^{2}(z) \mathbf{d} z^{2}$, more precisely:

Proposition 5.1. $\mathbf{V}(f, h, d)$ is a natural algebraic representation formula of type $(1,1,1)$ for curves of arbitrary arc length function $d$, i.e., the transformation rule $\mathbf{V}(f, h, d) \circ t=\mathbf{V}\left(f \circ t,(h \circ t) / t^{\prime}, t^{\prime}(d \circ t)\right)$ holds for arbitrary holomorphic parameter transformations $t . \mathbf{V}(f, h, d)$ can be inverted by differential operators involving square roots.

Proof. The first part follows from the construction. Again we can refer to the Mathematica facilities that permit to prove naturality of $\mathbf{V}(f, h, d)$ by verifying the condition (2.1).

However, a more conceptual proof is obtained by following the lines of sections 2 and 3. One defines a natural version $\mathbf{V}_{\Sigma}(\mathbf{f}, \mathbf{h}, \omega)$ of $\mathbf{V}(f, h, d)$, i.e., a family of operators $\mathbf{V}_{\Sigma}: \mathcal{A}_{\Sigma} \times \mathcal{X}_{\Sigma} \times \mathcal{L}_{\Sigma} \rightarrow \mathcal{A}_{\Sigma} \times \mathcal{A}_{\Sigma} \times \mathcal{A}_{\Sigma}$ for arbitrary Riemann surfaces $\Sigma$, whose local versions with respect to any holomorphic chart on $\Sigma$ agree with $\mathbf{V}(f, h, d)$. One observes that the derivative $\mathbf{W}^{\prime *}$ of the Weierstraß formula (4.5) for minimal curves in canonical parametrization is the local version of a natural operator $\mathbf{W}_{\Sigma}^{\prime *}: \mathcal{A}_{\Sigma} \rightarrow \mathcal{X}_{\Sigma} \times \mathcal{X}_{\Sigma} \times \mathcal{X}_{\Sigma}$ given by $\mathbf{W}_{\Sigma}^{\prime *}(\mathbf{f})=\left(1-\mathbf{f}^{2}, i\left(1+\mathbf{f}^{2}\right), 2 \mathbf{f}\right) \partial \mathbf{f} / 2$, where, as in proposition $2.3, \partial \mathbf{f}$ denotes the vector field dual to $\mathbf{d f}$. In analogy to $(5.1) \mathbf{V}_{\Sigma}(\mathbf{f}, \mathbf{h}, \omega)$ is defined by $\mathbf{V}(\mathbf{f}, \mathbf{h}, \omega)=$ $i \mathbf{V}^{(3)}(\mathbf{f}, \mathbf{h})+\left[\omega, \mathbf{W}_{\Sigma}^{\prime *}(\mathbf{f})\right]$, where $[.,]:. \mathcal{L}_{\Sigma} \times(\mathcal{X} \times \mathcal{X} \times \mathcal{X}) \rightarrow \mathcal{A} \times \mathcal{A} \times \mathcal{A}$ denotes the pairing of meromorphic 1-forms with triples of meromorphic vector fields.

The operator $\mathbf{V}_{\Sigma}$ maps triples $(\mathbf{f}, \mathbf{h}, \omega) \in \mathcal{A} \times \mathcal{X} \times \mathcal{L}$ to meromorphic curves in $\mathbb{C}^{3}$ whose infinitesimal arc length is the given meromorphic 1-form $\omega \in \mathcal{L}_{\Sigma}$. It has properties of injectivity and equivariance similar to those of $\mathbf{V}^{(3)}$ and $\mathbf{V}$. For the inverse operator $\mathbf{V}^{-1}$ see [6]. We describe it here in short for the convenience of the reader: Consider a curve $\Phi \in \mathcal{A}_{\mathbb{C}} \times \mathcal{A}_{\mathbb{C}} \times \mathcal{A}_{\mathbb{C}}$ with components $\Phi=\left(\Phi_{1}, \Phi_{3}, \Phi_{3}\right)$ and its preimage $\mathbf{V}^{-1}(\Phi)=$ $(f, h, \omega) \in \mathcal{A}_{\mathbb{C}} \times \mathcal{X}_{\mathbb{C}} \times \mathcal{L}_{\mathbb{C}}$. At first, we have $\omega=\sqrt{\left\langle\Phi^{\prime}, \Phi^{\prime}\right\rangle}$. Next, $f$ can be computed by $f=\left(\Phi_{3}^{\prime}-i \omega\right) /\left(\Phi_{1}^{\prime}-i \Phi_{2}^{\prime}\right)$. Finally, $h$ is given by $h=\left\langle\mathbf{W}_{\mathbb{C}}^{\prime *}, \Phi\right\rangle$ 
If $d \neq 0$ then for the curve $\mathbf{V}(f, h, d)$ the classical invariants 'curvature' and 'torsion' can be defined by the same analytic expressions as in the case of curves in $\mathbb{R}^{3}$. We are going to show that there is a differential algebraic relation between curvature and torsion of $\mathbf{V}(f, h, d)$ and the invariants 'canonical parameter' and 'minimal curvature' of the minimal curve $\mathbf{V}(f, h)$. These can be mutually expressed by differential operators.

For this purpose denote by $\mathcal{C}_{\Sigma}$ the vector space of all parametrized meromorphic curves in $\mathbb{C}^{3}$ defined on a Riemann surface $\Sigma$ and by $\mathcal{C}_{\Sigma, d} \subset \mathcal{C}_{\Sigma}$ the subset of curves with infinitesimal arc length $d \in \mathcal{A}_{\Sigma}$. We have $\operatorname{Min}_{\Sigma}=\mathcal{C}_{\Sigma, 0}$.

Denote by $\mathcal{L}_{\Sigma}^{*} \subset \mathcal{L}_{\Sigma}$ the set of all nonzero differential 1-forms on $\Sigma$. On the subset $\mathcal{C}_{\Sigma}^{*}=\mathcal{C}_{\Sigma} \backslash \operatorname{Min}_{\Sigma}$ consider the mapping that assigns to a curve $\Psi \in \mathcal{C}_{\Sigma}^{*}$ the triple $(\widetilde{\kappa}, \widetilde{\tau}, d) \in$ $\mathcal{A}_{\Sigma}^{2} \times \mathcal{L}_{\Sigma}^{*}$ consisting of its curvature $\widetilde{\kappa}(z)$, its torsion $\widetilde{\tau}(z)$ and its infinitesimal arc length $d(z)$. As in the case of curves in real Euclidean space $\mathbb{R}^{3}$ this mapping is bijective up to the choice of initial conditions for the solution of the system of Frenet equations. Similarly, let $(\kappa, \pi)$ be the mapping assigning to a curve $\Phi \in \operatorname{Min}_{\Sigma}$ the minimal curvature $\kappa(z)$ and the derivative $\pi(z)$ of its canonical parameter. In the same way as $(\widetilde{\kappa}, \widetilde{\tau}, d)$, the minimal curve is determined by $\kappa(z)$ and $\pi(z)$ (see [5]). Therefore there must exist a bijective mapping $\Omega$ that makes the following diagram of differential operators commutative.

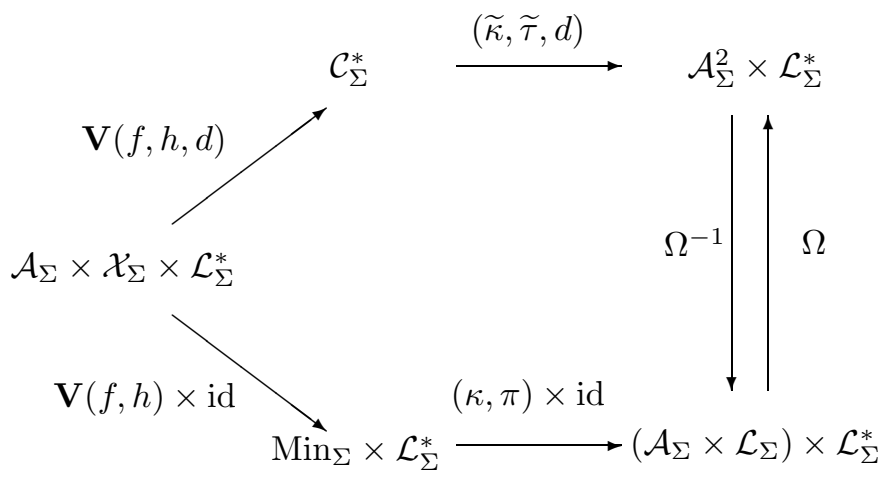

The mappings $\Omega$ and $\Omega^{-1}$ defined in this way are given by (5.3) and (5.4) below respectively. More precisely, $\Omega$ maps the direct factor $\mathcal{L}_{\Sigma}^{*}$ onto itself by the identity and $\mathcal{A}_{\Sigma} \times \mathcal{L}_{\Sigma}$ to $\mathcal{A}_{\Sigma} \times \mathcal{A}_{\Sigma}$ by (5.4). Similarly, $\Omega^{-1}$ acts also on $\mathcal{L}_{\Sigma}^{*}$ by the identity and maps $\mathcal{A}_{\Sigma}^{2}$ to $\mathcal{A}_{\Sigma} \times \mathcal{L}_{\Sigma}$ by $(5.5)$.

THEOREM 5.1. The curvature $\widetilde{\kappa}(z)$ and torsion $\widetilde{\tau}(z)$ of $\mathbf{V}(f, h, d)$ can be expressed as differential operators in the Study parameter $\kappa_{\Phi}$ of the minimal curve $\Phi=\mathbf{W}_{f}^{*}$ and the canonical parameter $\pi$ of the variation $\mathbf{V}(f, h)$ by

$$
\left\{\begin{aligned}
\widetilde{\kappa}^{2}= & \frac{d^{2} \kappa_{\Phi}^{2}-\pi^{4}-4 \pi^{2} d^{\prime}-3 d^{\prime 2}+4 d \pi \pi^{\prime}+2 d d^{\prime \prime}}{d^{4}} \text { and } \\
\widetilde{\tau}= & \frac{1}{\widetilde{\kappa}_{\Phi}^{2} d^{6}}\left\{\pi^{6}+6 \pi^{4} d^{\prime}+11 \pi^{2} d^{\prime 2}+6 d^{\prime 3}+d^{3} \kappa_{\Phi} \kappa_{\Phi}^{\prime}\right. \\
& -2 d\left(3 \pi^{3} \pi^{\prime}+5 \pi d^{\prime} \pi+2 \pi^{2} d^{\prime \prime}+3 d^{\prime} d^{\prime \prime}\right)+ \\
& \left.d^{2}\left(2 \pi^{\prime 2}+2 \pi \pi^{\prime \prime}+d^{(3)}-\kappa_{\Phi}^{2}\left(\pi^{2}+d^{\prime}\right)\right)\right\} .
\end{aligned}\right.
$$


The mapping $\left(\kappa_{\Phi}, \pi\right) \rightarrow\left(\widetilde{\kappa}^{2}, \widetilde{\tau}\right)$ is inverted by another differential operator, namely by

$$
\left\{\begin{aligned}
\kappa_{\Phi}= & d^{2} \widetilde{\kappa}^{2}+d^{2} \widetilde{\tau}^{2}+\frac{3 d^{\prime 2}}{d^{2}}-\frac{d \widetilde{\tau} \widetilde{\kappa}^{\prime 2}}{\widetilde{\kappa}^{2}}+\frac{d^{\prime} \widetilde{\kappa}^{\prime 2}}{d \widetilde{\kappa}^{2}}+\frac{5 \widetilde{\kappa}^{4}}{4 \widetilde{\kappa}^{4}}+ \\
& 2 d \widetilde{\tau}^{\prime}-\frac{2 d^{\prime \prime}}{d}-\frac{\widetilde{\kappa}^{\prime \prime 2}}{\widetilde{\kappa}^{2}}, \\
\pi= & \frac{d}{2}\left(-2 d \widetilde{\tau}+\frac{\widetilde{\kappa}^{\prime 2}}{\widetilde{\kappa}^{2}}\right) .
\end{aligned}\right.
$$

We refer the reader to a computer algebra system for a direct proof of these assertions.

The operator $\mathbf{V}(f, h, d)$ can be easily extended to a natural representation formula for minimal curves in $\mathbb{C}^{4}$ by replacing the function $d$ by its derivative $d^{\prime}$ and appending $i d(z)$ as a fourth component to the 3-dimensional vector $\mathbf{V}(f, h, d)$.

Simpler expressions for (5.3) and (5.4) are obtained if the argument $d$ in $\mathbf{V}(f, h, d)$ is replaced by $d(z)=-i d(z)$ and $h$ by $h(z)=-\mathbb{Q}_{f}^{(3)}\left(d^{\prime}(z) / f^{\prime}(z)\right)$, where $\mathbb{Q}_{f}^{(3)}$ is given by $(2.5)$ as the inverse operator of $\mathbb{P}_{f}^{(3)}$. The following results are given without proof. They come from computations with the computer algebra facilities of Mathematica.

Proposition 5.2. The two operators $\tilde{\mathbf{V}}$ and $\mathbf{W}^{(2)}$ given by

$$
\tilde{\mathbf{V}}(f, d)=\mathbf{V}\left(f,-\mathbb{Q}_{f}^{(3)}\left(\frac{d^{\prime}}{f^{\prime}}\right),-i d(z)\right) \text { and } \mathbf{W}^{(2)}(f, d)=\int d(\zeta) \frac{\mathbf{d}^{2} \mathbf{W}_{f}^{*}(\zeta)}{\mathbf{d} \zeta^{2}} \mathbf{d} \zeta
$$

assigning to a pair $(f, d)$ of meromorphic functions a meromorphic curve in $\mathbb{C}^{3}$ are identical, up to a constant vector of $\mathbb{C}^{3}$.

In the case $d \neq 0$, the curvature $\widetilde{\kappa}$ und torsion $\widetilde{\tau}$ of $\widetilde{\mathbf{V}}(f, d)$ are given by

$$
\widetilde{\kappa}(z)=-\frac{\kappa_{\Phi}(z)}{d(z)} \text { and } \widetilde{\tau}=\frac{\kappa_{\Phi}^{\prime}(z)}{d(z) \kappa_{\Phi}(z)},
$$

where $\kappa_{\Phi}(z)$ is the Study parameter of the minimal curve $\Phi=\mathbf{W}_{f}^{*}$.

The image of the operator $\widetilde{\mathbf{V}}$ consists of curves whose infinitesimal arc length function $d$, curvature $\widetilde{\kappa}$ und torsion $\widetilde{\tau}$ are subject to the condition

$$
i d(z)^{2} \widetilde{\tau}(z)+d^{\prime}(z)+d(z) \frac{\widetilde{\kappa}^{\prime}(z)}{\widetilde{\kappa}(z)}=0 .
$$

We solve the second of the equations (5.7) for $d(z)$ and insert the resulting expression as argument in $\tilde{\mathbf{V}}$. This gives a representation formula

$$
\overline{\mathbf{V}}(f, \widetilde{\tau})(z)=\widetilde{\mathbf{V}}\left(f, \frac{1}{\widetilde{\tau}} \frac{\mathbf{d} \log \left(\kappa_{\Phi}(z)\right)}{\mathbf{d} z}\right)
$$

for curves with preset torsion $\widetilde{\tau}$. The infinitesimal arc length $d s^{2}(z)$, curvature $\widetilde{\kappa}(z)$ and torsion $\widetilde{\tau}(z)$ of this curve also satisfy (5.8. One has

$$
d s^{2}(z)=-\frac{\kappa_{\Phi}^{\prime 2}(z)}{\kappa_{\Phi}^{2}(z) \widetilde{\tau}^{2}(z)} \text { and } \widetilde{\kappa}(z)=\frac{\kappa_{\Phi}(z)^{2} \widetilde{\tau}(z)}{\kappa_{\Phi}^{\prime}(z)}
$$

6. Algebraic representation formulas obtained from caustics. A surface strip in $\mathbb{R}^{3}$ is a pair $(\beta(t), \gamma(t))$ of two parametrized curves such that $\|\gamma(t)\|=1$ and $\left\langle\beta^{\prime}(t), \gamma(t)\right\rangle$ $=0$. One can imagine $\beta$ as a curve on a surface $\mathcal{F} \subset \mathbb{R}^{3}$ and $\gamma$ the restriction of the normal 
vector field of $\mathcal{F}$ to $\beta$. In this case we say that $\mathcal{F}$ is a surface passing through the strip $(\beta, \gamma)$.

By a classical result of $\mathrm{H}$. A. Schwarz, through any analytic strip $(\beta(t), \gamma(t))$ passes a unique minimal surface given by the real part of the minimal curve

$$
\mathcal{B}_{\beta, \gamma}(z)=\beta(z)-i \int_{z_{0}}^{z} \beta^{\prime}(\zeta) \times \gamma(\zeta) \mathbf{d} \zeta
$$

where $\times$ is the ordinary vector product extended to $\mathbb{C}^{3}$ and $\beta(\zeta), \gamma(\zeta)$ are holomorphic extensions of $\beta$ and $\gamma$ to $\mathbb{C}$. This is a (nonalgebraic) representation formula named after E. G. Björling, who was the first to pose the problem of determining all minimal surfaces passing through a given strip.

If $a(t), b(t)$ are analytic functions, $\beta(t)=(a(t), b(t))$ the planar analytic curve defined by them and $\gamma(t)=\left(-b^{\prime}(t), a^{\prime}(t)\right) / \sqrt{\left.\left.a^{\prime 2}(t)\right)+b^{\prime 2}(t)\right)}$ its normal vector field, then (6.1) applied to the plane curves $\beta$ and $\gamma$ (that have to be considered as space curves under the embedding $\mathbb{R}^{2} \subset \mathbb{R}^{3}$ ) yields the local representation formula

$$
\widetilde{\mathcal{B}}_{\beta}(z)=\left(a(z), b(z),-i \int_{z_{0}}^{z} \sqrt{\left(\left(a^{\prime}(\zeta)\right)^{2}+\left(b^{\prime}(\zeta)\right)^{2}\right.} \mathbf{d} \zeta\right),
$$

that is not algebraic. However, it becomes algebraic when combined with several operations of forming envelopes of families of straight lines, mainly caustics of planar curves.

Let $\delta_{t}(v)=\alpha(t)+v \varepsilon(t)$ be a 1-parameter family of straight lines parametrized by a planar base curve $\alpha(t)=(x(t), y(t))$ and a director curve $\varepsilon(t)=(\xi(t), \eta(t))$. The envelope of the family $\delta$ is the curve

$$
\beta(t)=\alpha(t)+\frac{\eta(t) x^{\prime}(t)-\xi(t) y^{\prime}(t)}{\xi(t) \eta^{\prime}(t)-\eta(t) \xi^{\prime}(t)} \varepsilon(t)
$$

We consider the following special cases:

evolute - this is the envelope of the family of normal lines of $\alpha$, i.e., the evolute is obtained by putting $\varepsilon(t)=\left(-y^{\prime}(t), x^{\prime}(t)\right)$ in $(6.3)$. We obtain $\beta(t)=\alpha(t)+\nu(t) / \kappa(t)$, where $\nu$ and $\kappa$ are the oriented normal vector and the signed curvature respectively. central caustic - the envelope of the family of light rays emanating from a center $P=\left(p_{x}, p_{y}\right) \in \mathbb{R}^{2}$, the light source, after reflection at the curve $\alpha(t)$. In this case $\varepsilon(t)$ is the vector

$$
\varepsilon(t)=\gamma(t)-2 f \frac{\left\langle\gamma(t), \alpha^{\prime}(t)\right\rangle}{a^{\prime 2}(t)+b^{\prime 2}(t)} \alpha^{\prime}(t), \text { where } \gamma(t)=\alpha \overrightarrow{(t)} P
$$

parallel caustic - the envelope of the family of parallel light rays after reflection at the curve $\alpha$. We assume that the incoming light rays are parallel to the $x$-axis. The explicit formula for the parallel caustic comes out by putting $\varepsilon(t)=(\xi(t), \eta(t))=$ $\left(x^{\prime}(t)^{2}-y^{\prime}(t)^{2}, 2 x^{\prime}(t) y^{\prime}(t)\right)$ in (6.3). This is the limiting case of central caustic for $p_{x} \rightarrow \infty$.

These 3 operations are more or less involved nonlinear differential operators in $x(t)$ and $y(t)$. We will see that they give ARF's if we consider them as operators mapping planar curves to planar curves and compose them with the Björling formula (6.2). The essential result will be that the arc length function 


$$
s_{x, y}(t)=\int_{t_{0}}^{t} \sqrt{a^{\prime 2}(\tau)+b^{\prime 2}(\tau)} \mathbf{d} \tau
$$

figuring in (6.2) is an algebraic expression involving the components $x(t)$ and $y(t)$ of the curve $\alpha(t)$ when $a(\tau)$ and $b(\tau)$ are the components of the evolute, the central caustic or the parallel caustic of $\alpha$ respectively. We have explicitly:

Proposition 6.1. Let $\alpha(t)$ be a planar curve in analytic parametrization $(x(t), y(t))$,

$$
\kappa=\frac{x^{\prime} y^{\prime \prime}-y^{\prime} x^{\prime \prime}}{\left\langle\alpha^{\prime}, \alpha^{\prime}\right\rangle^{3 / 2}} \text { and } \nu=\frac{1}{\sqrt{\left\langle\alpha^{\prime}, \alpha^{\prime}\right\rangle}}\left(\begin{array}{c}
-y^{\prime} \\
x^{\prime}
\end{array}\right)
$$

the signed curvature and the oriented unit normal vector of $\alpha(t)$ respectively. Let $\beta(t)=$ $(a(t), b(t))$ be the analytic parametrization of any of the three types 'evolute', 'central caustic' and 'parallel caustic' of envelopes of families of lines associated with $\alpha$. Up to an additive constant the arc length function of $\beta$ is given in terms of the functions $x(t), y(t)$ and its derivatives by the following table:

\begin{tabular}{|c|c|}
\hline type of envelope & $\begin{array}{c}\text { arc length function } \\
\int\left\|\alpha^{\prime}(\tau)\right\| \mathbf{d} \tau\end{array}$ \\
\hline \hline evolute & $\frac{1}{\kappa(t)}$ \\
\hline $\begin{array}{c}\text { central caustic } \\
\text { with light source }(0,0)\end{array}$ & $-\left(\frac{1}{\|\alpha(t)\|}+\frac{\langle\alpha(t), \nu(t)\rangle}{2\|\alpha(t)\|^{3} \kappa(t)}\right)^{-1}$ \\
\hline parallel caustic & $a(t)+\frac{b^{\prime}(t)}{2 \kappa(t) \sqrt{a^{\prime 2}(t)+b^{\prime 2}(t)}}$ \\
\hline
\end{tabular}

Proof. The first assertion is due to the fact that the constructions of the evolute and the involute of a curve are inverse to each other. We are not going to derive the two other results from geometric facts in a similar way and recommend to verify them with the help of a computer algebra system. In order to prove for instance the second assertion with Mathematica, one could start by encoding the formula

$$
\frac{2}{n}\left(\begin{array}{c}
2 x y x^{\prime} y^{\prime 2}-y^{2}\left({x^{\prime}}^{2} y^{\prime}+x y^{\prime} x^{\prime \prime}-x x^{\prime} y^{\prime \prime}\right)-x^{2}\left(y^{\prime 3}+x y^{\prime} x^{\prime \prime}-x x^{\prime} y^{\prime \prime}\right) \\
y^{2} x^{\prime 3}+x^{2} x^{\prime} y^{\prime 2}+y^{3}\left(x^{\prime} y^{\prime \prime}-y^{\prime} x^{\prime \prime}\right)+x y\left(x x^{\prime} y^{\prime \prime}-2 x^{\prime 2} y^{\prime}-x y^{\prime} x^{\prime \prime}\right)
\end{array}\right),
$$

where $n=y{x^{\prime}}^{3}-x{x^{\prime}}^{2} y^{\prime}+y x^{\prime}{y^{\prime}}^{2}-x y^{\prime 3}-2 x^{2} y^{\prime} x^{\prime \prime}-2 y^{2} y^{\prime} x^{\prime \prime}+2 x^{2} x^{\prime} y^{\prime \prime}+2 y^{2} x^{\prime} y^{\prime \prime}$, for the central caustic of $\alpha(t)$ as a Mathematica command, called for instance centralcaustic [alpha] [t]. Similarly, a command kappa2 [alpha] [t] for the curvature of a planar curve (see [7]) is needed. Applying the following instructions to Mathematica yields 0 .

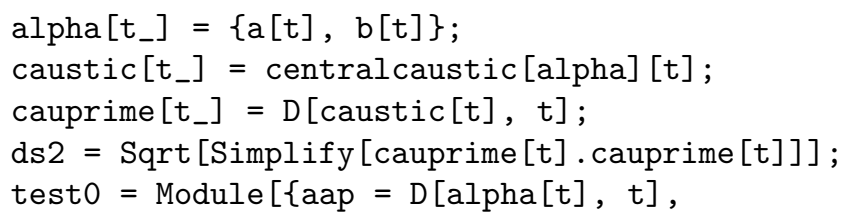




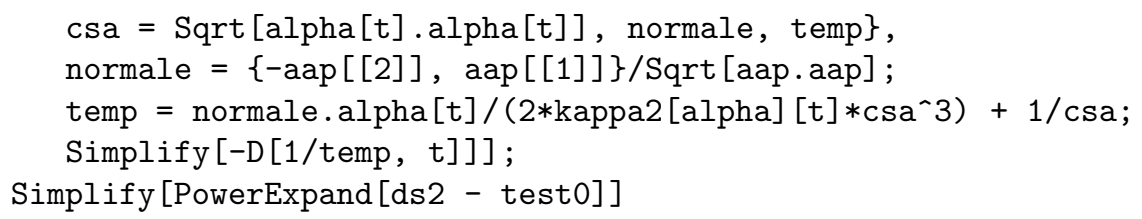

7. Graphical examples. The helicoid is given in the canonical Weierstraß representation $\mathbf{W}^{*}(f)(z)$ by functions of the form $f(z)=e^{a z}$, where $a \in \mathbb{R}$ is a non-zero constant. The first 4 pictures show two examples of variations and deformations of a helicoid with elementary functions. The first with the function $h(z)=e^{3 z}$ and the second with $h(z)=z^{2} e^{z / 10}$. These surfaces can be defined by a simple Mathematica command using the operator $\mathrm{OP}[\mathrm{n}][\mathrm{f}, \mathrm{h}][\mathrm{z}]$ defined in section 2 together with an implementation of the Weierstraß formulae (2.2) and (4.5). The commands

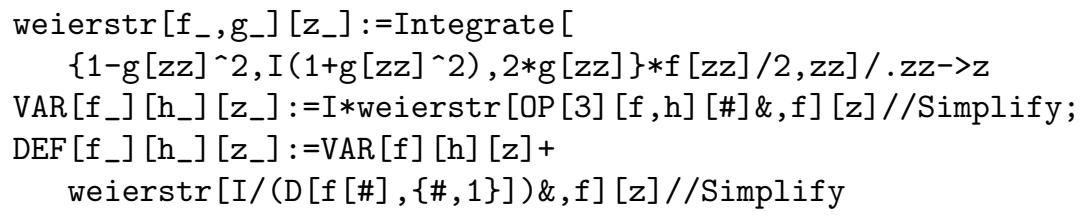

are a quick way to define the variation and deformation operators of section 4 in Mathematica.

The associated surface given as real part of a minimal curve $\Phi(u+i v)$ is the imaginary part of $\Phi(u+i v)$. The next 4 pictures show two surfaces obtained from the deformations and variations of the minimal curve

$$
\Phi(z)=\left(\frac{i}{4} \sin (2 z), \frac{-z}{2},-\frac{i}{2} \cos ^{2}(z)\right)^{\top}
$$

with certain functions $h(z)$ together with their associated surfaces. These deformations and variations are computed directly with the formula (4.3) of proposition 4.1. If the surface parameter $v$ is near to 0 the corresponding part of the surface appears as a localized distortion of the helicoid or the catenoid respectively.

If we define a Mathematica command centralcaustictominimalcurve encoding the formula of proposition 6.1 , we can study minimal surfaces that intersect the $x y$-plane along central caustics of circles or ellipses. We present the caustic of the circle generated by the light rays emanating from the center $P=(19 / 11,0)$. The Björling curve associated to this caustic is produced with

centralcaustictominimalcurve $[\{\operatorname{Cos}[\#], \operatorname{Sin}[\#]\} \&, 19 / 11,0][z]$.

The result is

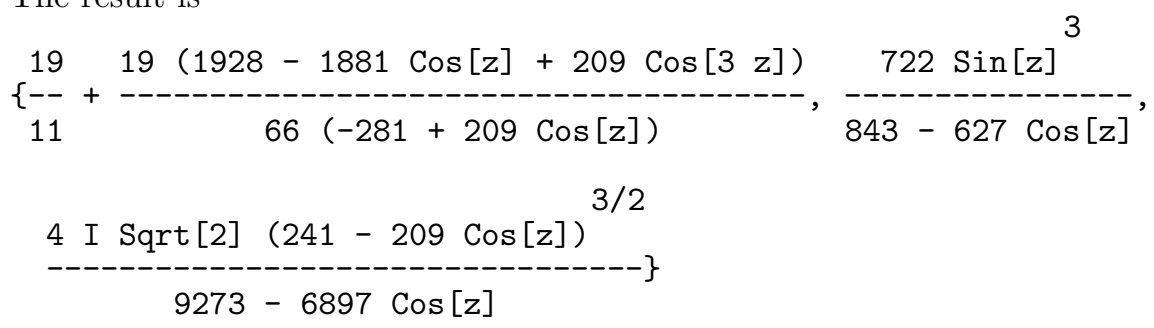


The pictures show the circle, the light rays emanating from $P$, the reflected rays, their enveloping caustic and the minimal surface associated to the caustic by the Björling formula (6.2). A second example shows the minimal surface of the caustic of the ellipse: The command

phi $\left[z_{-}\right]=$centralcaustictominimalcurve $[\{3 * \operatorname{Cos}[\#], 2 * \operatorname{Sin}[\#]\} \&, 3,-2][z]$ yields the minimal curve

$$
\Phi(z)=\frac{1}{\widetilde{\Phi}(z)}\left(\begin{array}{c}
198-369 \cos (z)+90 \cos (2 z)-15 \cos (3 z)+45 \sin (z)-63 \sin (3 z) \\
28-30 \cos (z)-60 \cos (2 z)+62 \cos (3 z)-66 \sin (z)-10 \sin (3 z) \\
-2 i \sqrt{2(39-36 \cos (z)+5 \cos (2 z)+16 \sin (z))^{3}}
\end{array}\right)
$$

where $\widetilde{\Phi}=-130+123 \cos (z)-30 \cos (2 z)+5 \cos (3 z)-33 \sin (z)-5 \sin (3 z)$.

The fourth pair of pictures shows at the top the ellipse with semi axes $a=3, b=2$, the light rays emanating from the point $(3,-2)$, the reflected light rays together with their caustic and on the bottom the minimal surface given by the minimal curve $\Phi$ above.

7.1. Deformation and variation of the helicoid by means of an exponential function. We put $f(z)=e^{4 z}$ and $h(z)=e^{3 z} / 7$. The Weierstraß formula applied to $f$ yields a helicoid. The first picture shows the deformation and the second the variation of this helicoid with the function $h$. Mathematica commands that produce these pictures are indicated.
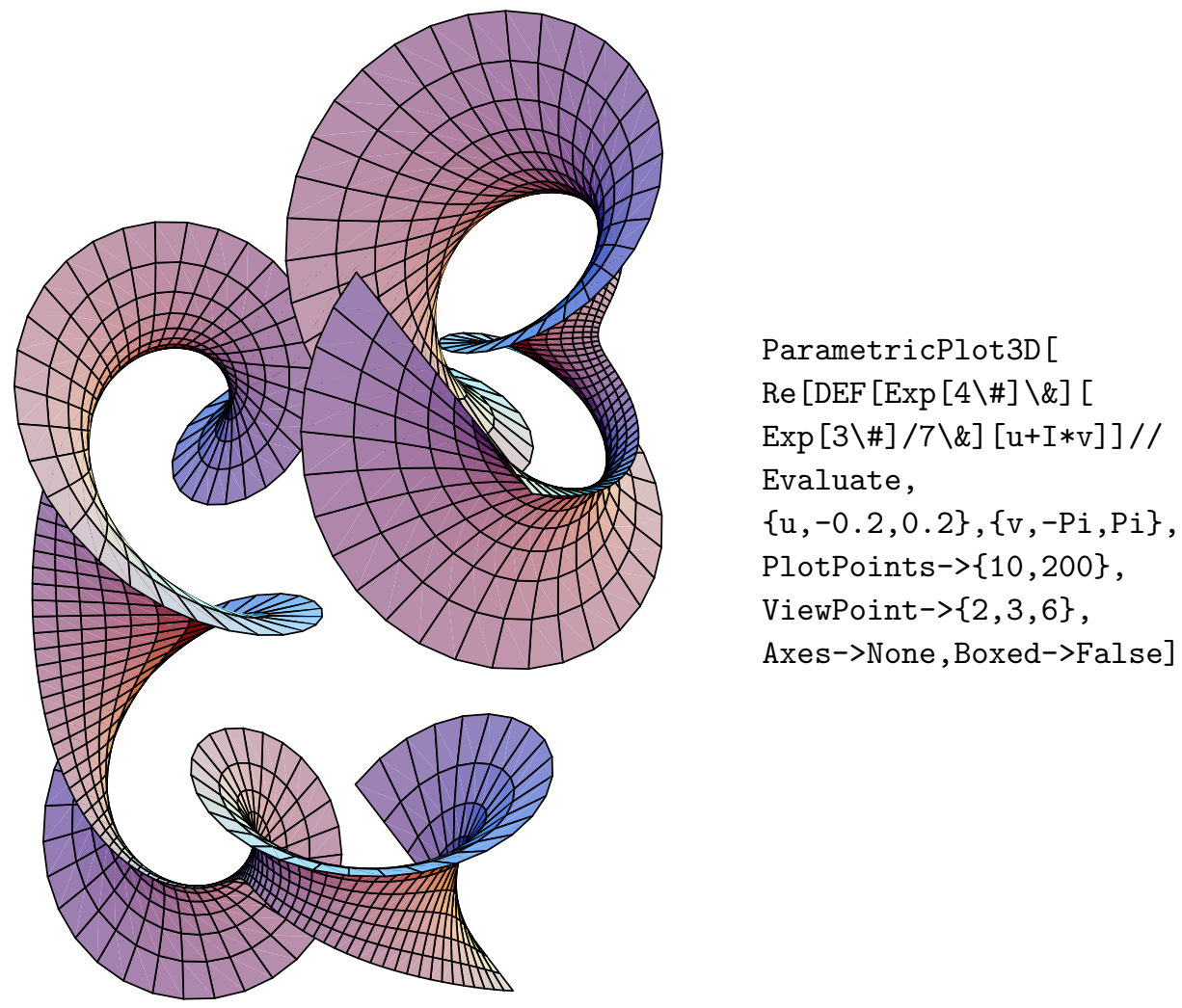


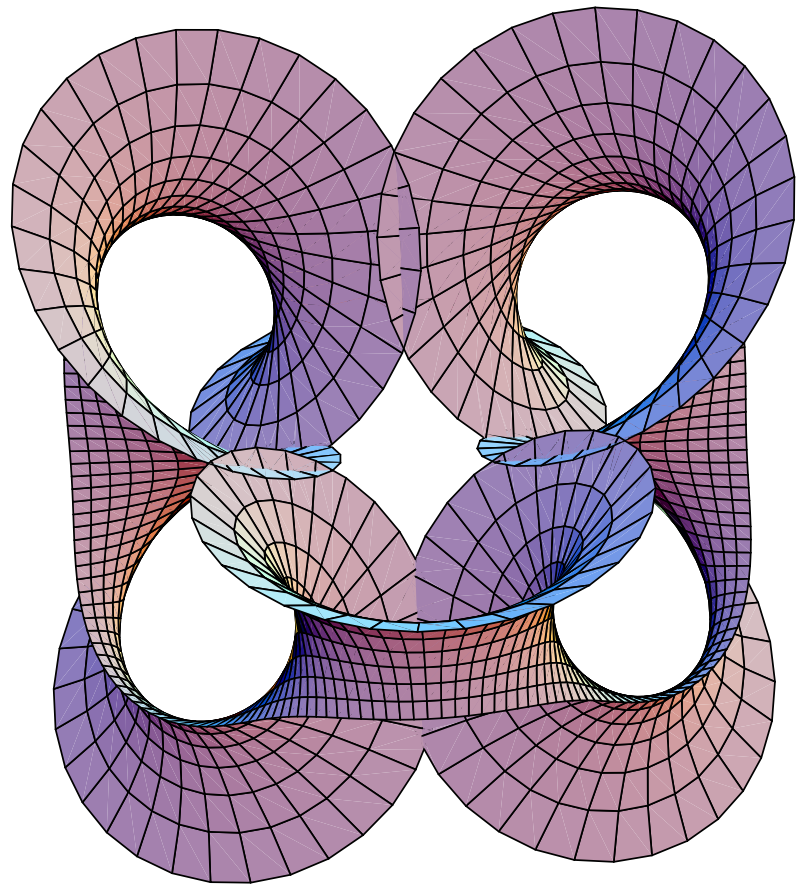

ParametricPlot3D [

$\operatorname{Re}[\operatorname{VAR}[\operatorname{Exp}[4 \backslash \#] \backslash \&][$

$\operatorname{Exp}[3 \backslash \#] / 7 \backslash \&][\mathrm{u}+\mathrm{I} * \mathrm{v}]] / /$

Evaluate,

$\{\mathrm{u},-0.2,0.2\},\{\mathrm{v},-\mathrm{Pi}, \mathrm{Pi}\}$, PlotPoints $->\{10,200\}$, ViewPoint- $>\{2,3,6\}$, Axes $->$ None,

Boxed->False] ;

7.2. Variation of the helicoid. The picture shows the variation of the surface $\Re\left(\mathbf{W}_{\exp (z)}^{*}(x+i y)\right)$ by means of the function $h(z)=z^{2} e^{z} / 10$.

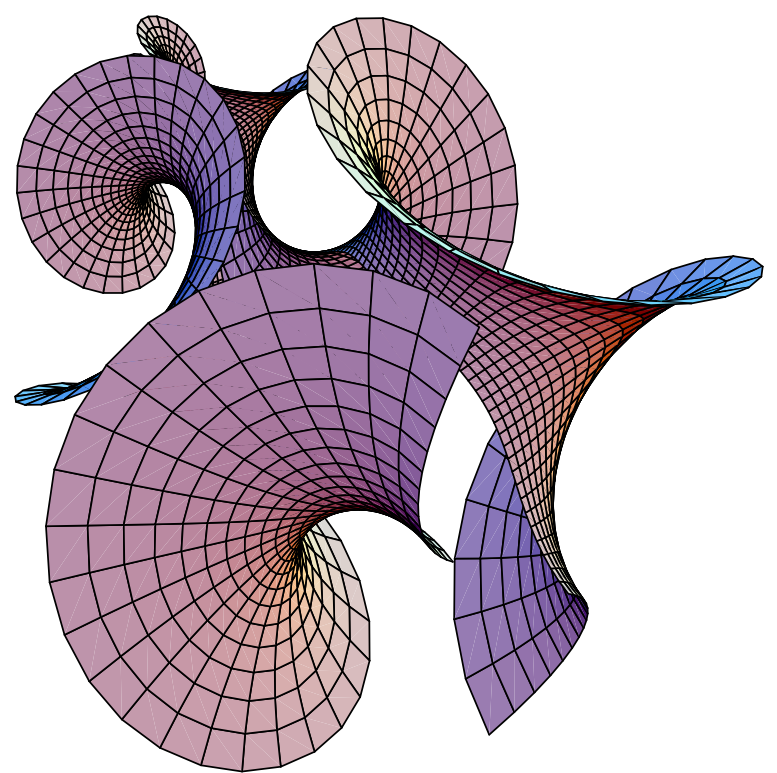

ParametricPlot3D [

$\operatorname{Re}\left[\operatorname{VAR}\left[\mathrm{E}^{\wedge} \#\right.\right.$ \&] $\left[\#^{\wedge} 2 * \mathrm{E}^{\wedge} \# / 10\right.$ \&] [ $\mathrm{u}+\mathrm{I} * \mathrm{v}]] / /$ Evaluate, $\{\mathrm{u},-0.7,0.7\},\{\mathrm{v},-4 \mathrm{Pi}, 4 \mathrm{Pi}\}$, PlotPoints $\rightarrow\{17,211\}$, ViewPoint $\rightarrow\{2,3,6\}$, Axes -> None, Boxed $\rightarrow$ False] 
130

H. GOLLEK

7.3. Deformations of the helicoid. The minimal curve of the helicoid can be defined with the function $f(z)=\tan (z)$ in Mathematic via DEF [Tan [\#] \&] [0\&] [z]. The two pictures show the surfaces whose minimal curves are DEF [Tan [\#] \&] [h] [z] for the functrons

$$
h(z)=\frac{(1+2 i)(1-\cos (z)}{z^{2}} \text { and } \quad h(z)=\frac{i \sin ^{6}(z)}{z^{6}} .
$$
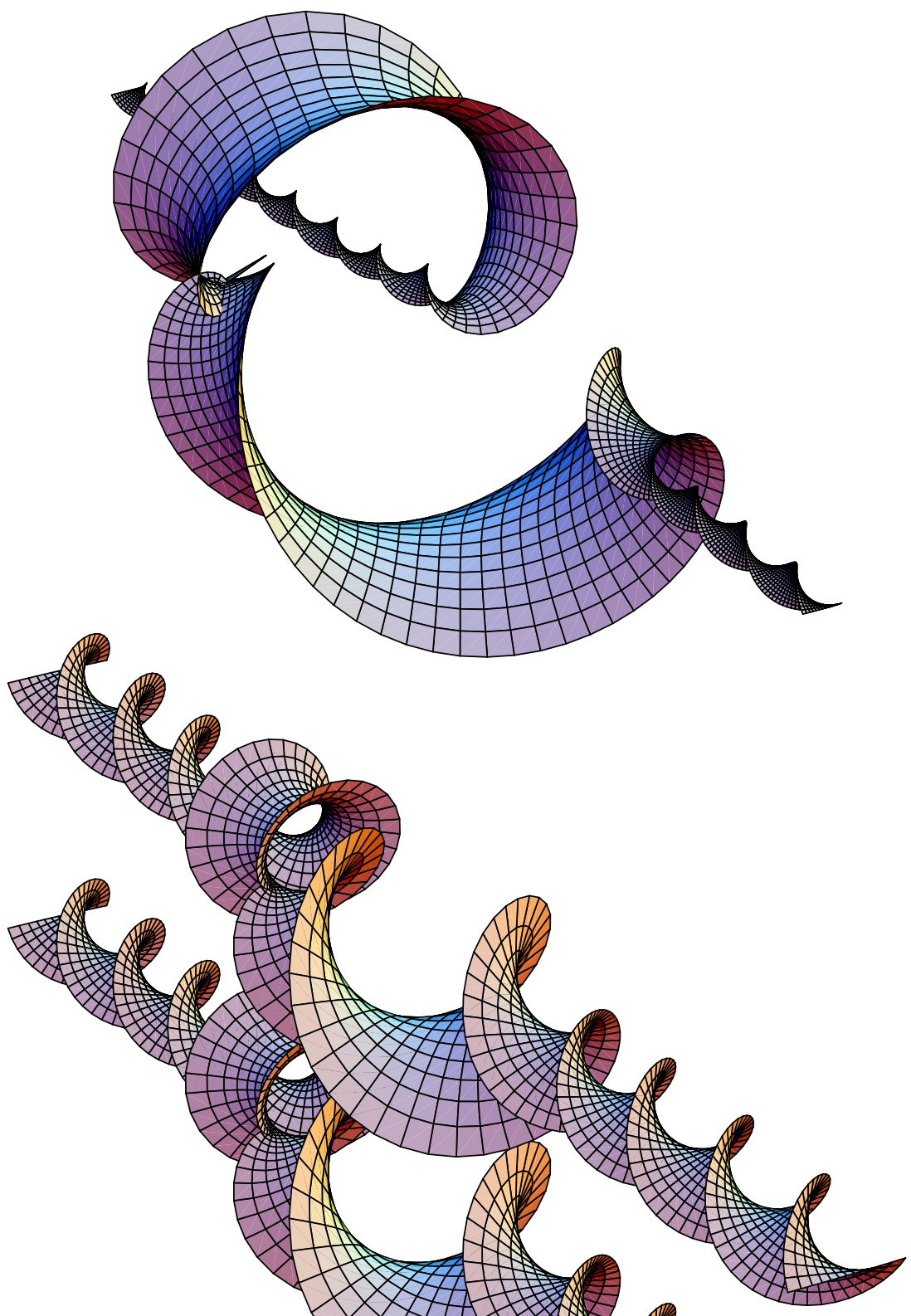
7.4. Deformations of the helicoid. We choose

$$
h(z)=\frac{(17+11 i) \sin ^{2}(z)}{z^{2}}
$$

and draw the deformation $\operatorname{Re}[\operatorname{DEF}[\operatorname{Tan}[\#] \&][\mathrm{h}][\mathrm{u}+\mathrm{i} * \mathrm{v}]]$ and its associated surface $\operatorname{Im}[\mathrm{DEF}[\operatorname{Tan}[\#] \&][\mathrm{h}][\mathrm{u}+\mathrm{i} * \mathrm{v}]]$. Observe that the associated surface has the appearance of a deformation of the catenoid.

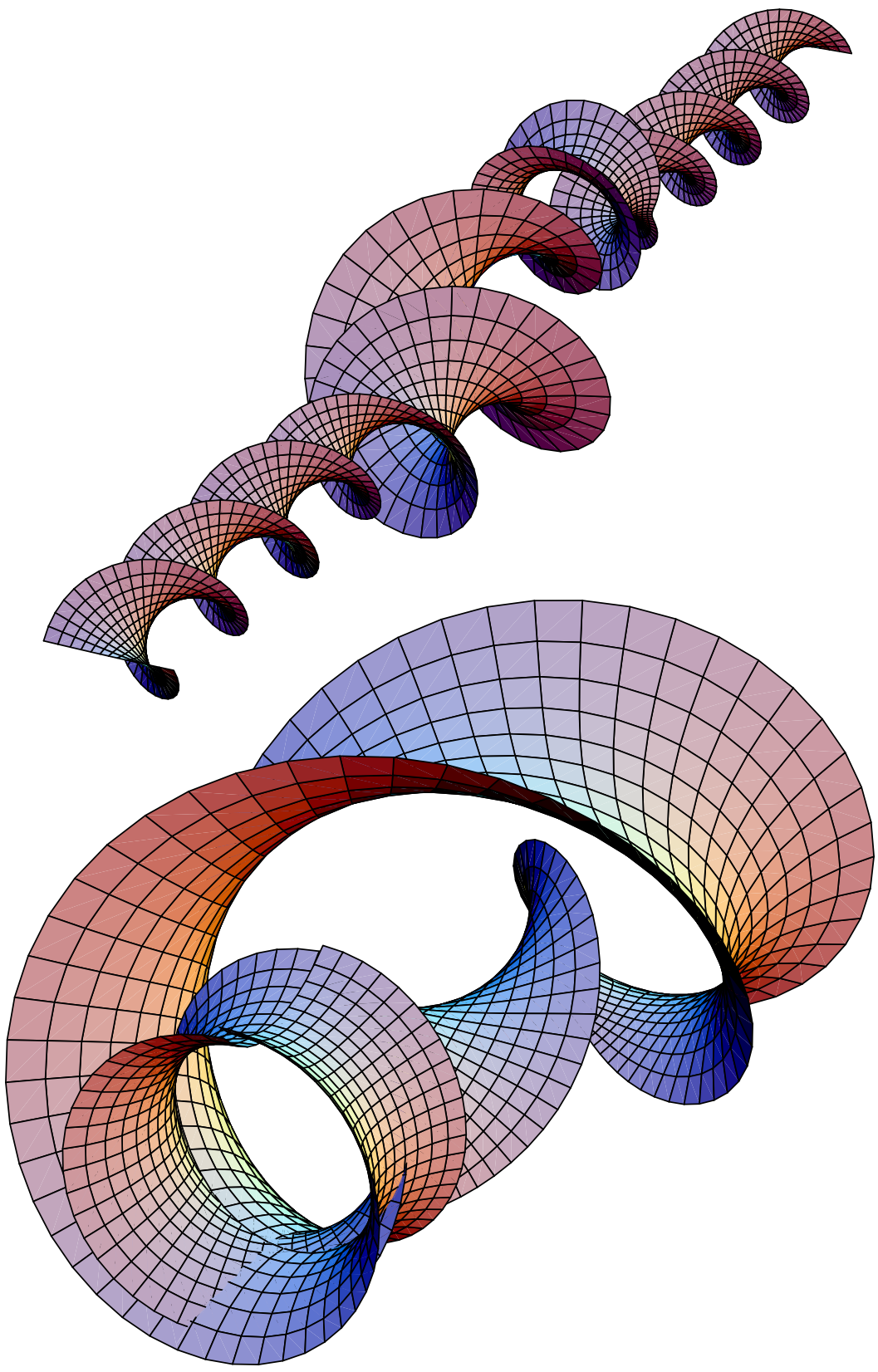


7.5. A caustic of a circle. The first plot shows the caustic of light rays emanating from a point on the $x$-axis and the second one a minimal surface intersecting the $x y$-plane in this caustic perpendicularly.
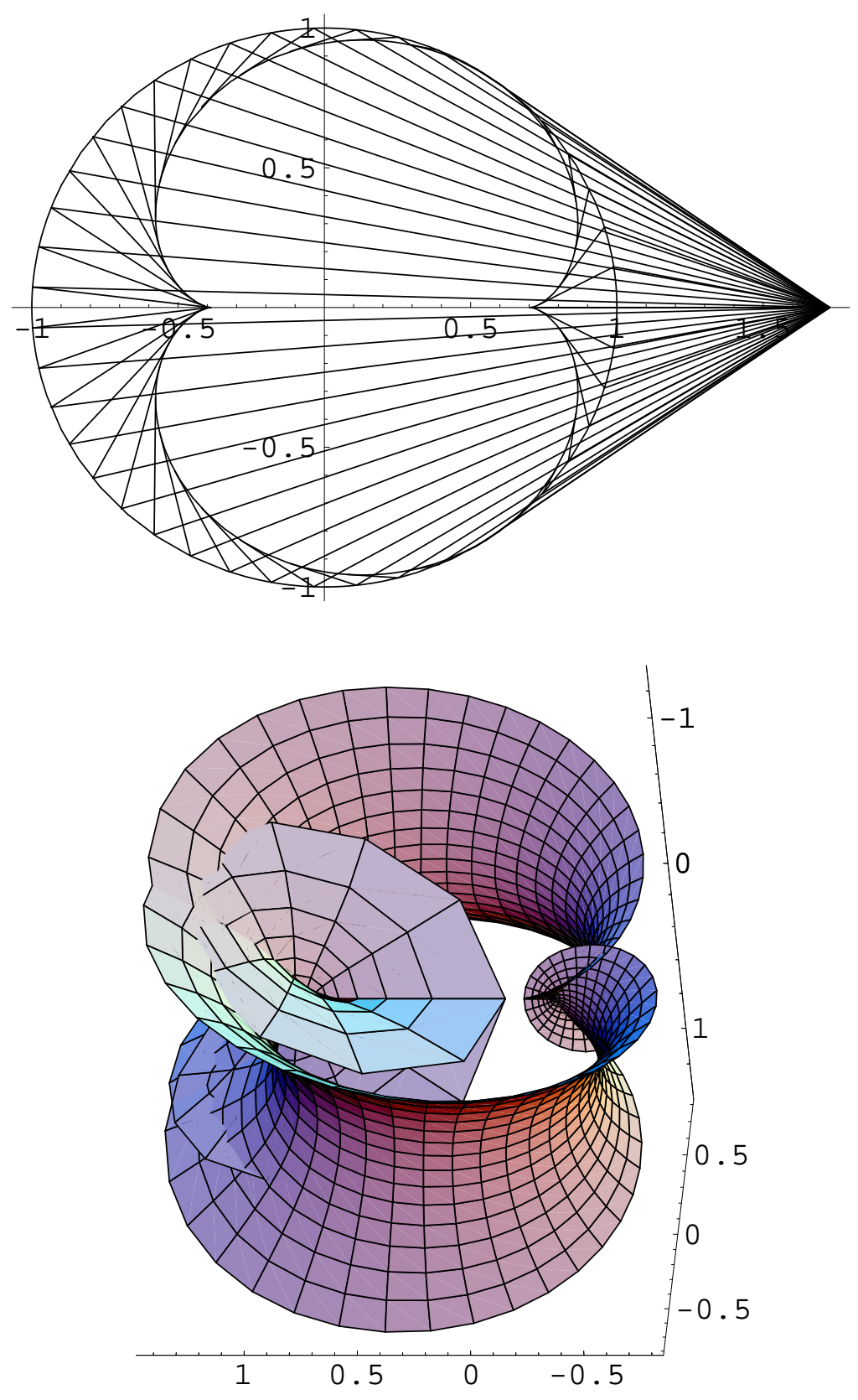
7.6. A caustic of an ellipse and the minimal surface intersecting the xy-plane in this caustic perpendicularly.
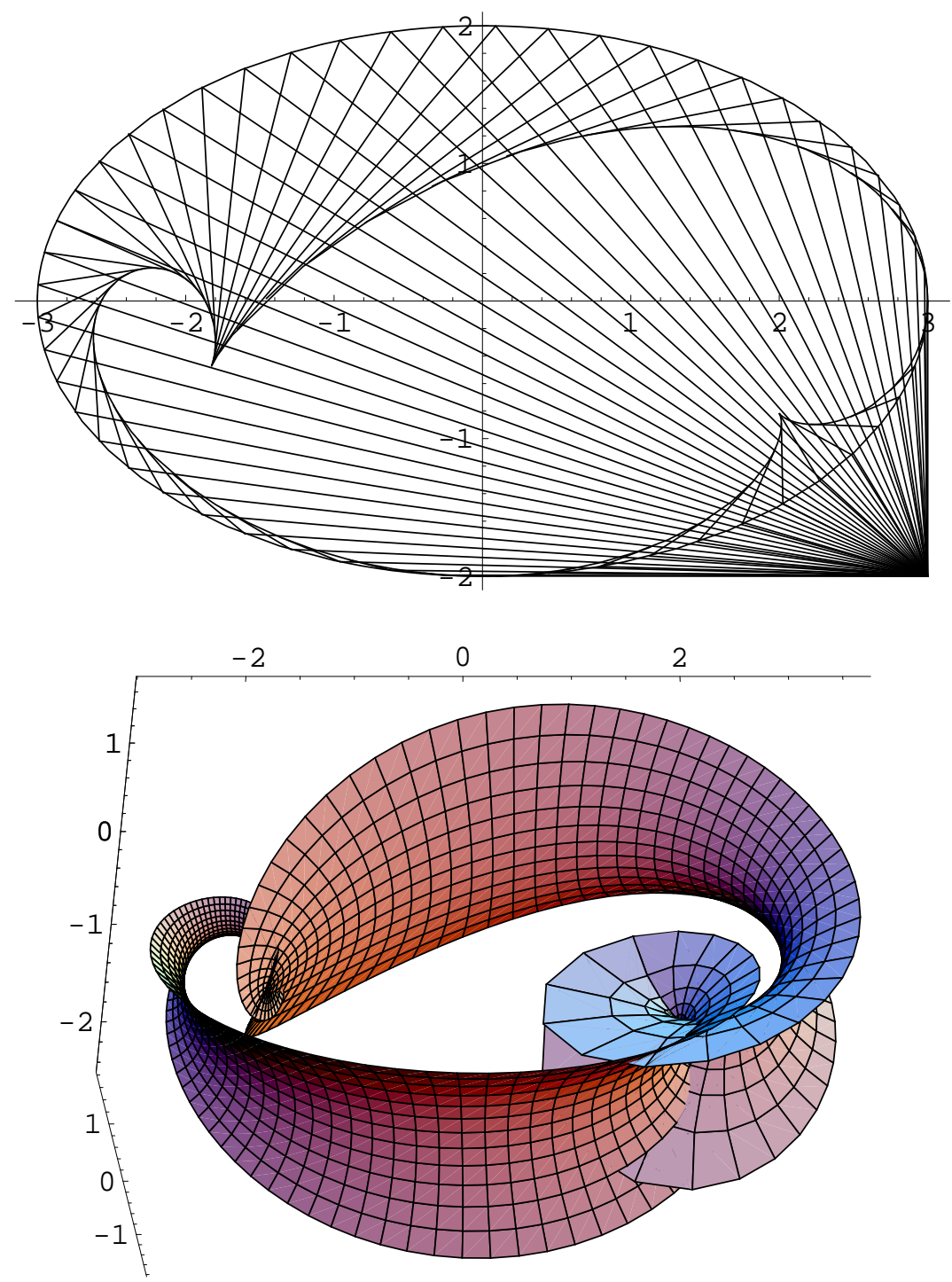

\section{References}

[1] W. Blaschke, Vorlesungen über Differentialgeometrie.Vol. I: Elementare Differentialgeometrie, 4-th ed., Springer, Berlin, 1945.

[2] R. Bryant, S. S. Chern, R. B. Gardner, H. L. Goldschmidt and P. A. Griffiths, Exterior Differential Systems, Springer, New York, 1991, 
[3] U. Dierkes, S. Hildebrandt, A. Küster and O. Wohlrab, Minimal Surfaces, Vol. 1, Springer, Berlin, 1992.

[4] H. Gollek, Natural Equations of minimal surfaces and the Weierstraß-representation formula, unpublished tutorial notes, containing Mathematica programs, examples and graphics; see: www-irm.mathematik.hu-berlin.de/ gollek/MinSurfs/.

[5] H. Gollek, Deformations of minimal curves in $\mathbb{C}^{3}$, in Proc: 1-st NOSONGE Conference, Warsaw, Sept. 1996.

[6] H. Gollek, Deformations of minimal Surfaces containing planar Geodesics, in: Proc. 18-th Winter School "Geometry and Physics", Srni (Czechoslovakia), 1998.

[7] A. Gray, Modern Differential Geometry of Curves and Surfaces, CRC Press, 1995.

[8] I. Kolár, P. W. Michor and J. Slovák, Natural Operations in Differential Geometry, Springer, 1993.

[9] J. C. C. Nitsche, Lectures on Minimal Surfaces, Vol. 1, Cambridge Univ. Press, Cambridge, 1989. English translation of the first part of: Vorlesungen über Minimalflächen, Grundlehren Math. Wiss. 199, Springer, Berlin, 1975.

[10] H. Pabel, Deformationen von Minimalflächen, Geometrie und ihre Anwendungen, Carl Hanser Verlag, München, 1994. 